\title{
Systematics of the multi-Regge three-loop symbol
}

\section{Till Bargheer}

DESY Theory Group, DESY Hamburg,

Notkestraße 85, D-22607 Hamburg, Germany

E-mail: till.bargheer@desy.de

ABStRACT: We review the systematics of Mandelstam cut contributions to planar scattering amplitudes in the multi-Regge limit. Isolating the relevant cut terms, we explain how the BFKL expansion can be used to construct the perturbative $n$-point multi-Regge limit amplitude in certain kinematic regions from a finite number of basic building blocks. At three loops and at leading logarithmic order, two building blocks are required. Their symbols are extracted from the known three-loop six-point and seven-point symbols for general kinematics. The new seven-point building block is constructed in terms of single-valued multiple polylogarithms to the extent it can be determined using the symbol as well as further symmetry and consistency constraints. Beyond the leading logarithmic order, the subleading and sub-subleading terms require two and one further building block, respectively. The latter could either be reconstructed from further perturbative data, or from BFKL integrals involving yet-unknown corrections to the central emission block.

Keywords: 1/N Expansion, Extended Supersymmetry, Scattering Amplitudes

ARXIV EPRINT: 1606.07640 


\section{Contents}

1 Introduction 1

2 Background 3

$\begin{array}{llr}3 & \text { Symbols and regions } & 9\end{array}$

4 Two-loop expansion $\quad 11$

5 Three-loop expansion $\quad 15$

$\begin{array}{llr}6 & \text { Building blocks } & 18\end{array}$

$\begin{array}{llr}7 & \text { The function } g_{3} & 20\end{array}$

$\begin{array}{lll}8 & \text { Conclusion } & 27\end{array}$

$\begin{array}{ll}\text { A Reduction identities } & \mathbf{2 9}\end{array}$

$\begin{array}{ll}\text { B Four-loop expansion } & 30\end{array}$

$\begin{array}{ll}\text { C Polylogarithm identities } & 33\end{array}$

D The function $g_{3}$ at LLA $\quad 34$

\section{Introduction}

Recent advances in the study of scattering amplitudes have sparked renewed interest in the multi-Regge limit of high-energy scattering. Besides its phenomenological significance, it has long been noted that the perturbative expansion simplifies considerably in this limit: typically, the perturbative series has to be (and in fact can be!) resummed due to the appearance of large logarithms, leading to factorized all-order expressions for scattering processes. A further enhancement comes about in the case of planar $\mathcal{N}=4$ super Yang-Mills theory: here, the multi-Reggeon states that resum all-order gluon exchanges are governed by the integrable Balitsky-Fadin-Kuraev-Lipatov (BFKL) [1-3] and Bartels-KwieciǹskiPraszałowicz (BKP) $[4,5]$ Hamiltonians. This first appearance of integrability in the planar theory was observed long before the extensive discoveries and applications of integrable structures that took place during the past fifteen years [6]. Since the proposal of the exponentiated Bern-Dixon-Smirnov (BDS) amplitude [7], the systematics of multi-Regge limit amplitudes in planar $\mathcal{N}=4$ super Yang-Mills theory have been understood to a remarkable extent. In fact, after a disagreement at strong coupling had casted doubt on the correctness 
of the BDS amplitude [8], it was the absence of the expected Regge pole and cut terms that invalidated the proposal at weak coupling [9], and that prompted the correction of the BDS amplitude by the dual conformally invariant remainder function beyond five points [10, 11].

By now, the remainder function has been constructed to high loop orders by constraining the possible function space through physical symmetry and analyticity requirements $[12,13]$. This bootstrap program relies on various input, ranging from the mathematical theory of the relevant functions $[14,15]$ to recursion relations [16] and the expansion around collinear limits as dictated by integrability $[17,18]$. In all cases, knowledge about the multi-Regge limit has provided important boundary data to the bootstrap enterprise. Conversely, these recent methods admit to compute the BFKL data, and hence the multiRegge-limit remainder function, to unprecedented orders [12, 19-23]. To date, this fruitful interplay has mostly been restricted to the six-point case. An extension to seven-point functions has been initiated recently [24]. Going to even higher points will require a better understanding of the relevant function space. It is conceivable that the Regge limit will again provide valuable boundary data in this regard.

It has been understood that obtaining the full analytic structure of multi-Regge limit amplitudes requires to analyze the amplitudes in all possible kinematic regions [25-28]. In fact, while the integrable structure at strong coupling becomes particularly amenable in the multi-Regge limit [29], a discrepancy with the expectation from weak coupling has been observed in one of the kinematic regions at seven points [30]. Recently, a systematic study of the $n$-point two-loop remainder function in all kinematic regions at weak coupling has been put forward [31]. The ability to study any number of points relied on the known two-loop symbol of the remainder function for all multiplicities [32]. Passing from polylogarithmic functions to their symbols constitutes a major simplification, both for the analysis of the relevant expressions and for the systematics of the multi-Regge limit.

The goal of the present work is two-fold: one aim is to understand the results of the previous study [31] from the perspective of Regge cut contributions. Secondly, we want to lift the analysis to the three-loop level. To this end, we first isolate the Regge cut contributions that contribute to a given region, and then expand the relevant contributions to the three-loop order. Judiciously grouping the resulting terms, we find that the $n$-point three-loop remainder function, in the simplest class of kinematic regions, reduces to a linear combination of five building block functions. At the symbol level, the reduction extends to all kinematic regions. The symbols of the two building blocks required to reconstruct the $n$-point remainder function at leading logarithmic order are extracted from the known perturbative data. To the extent that it is fixed by the symbol as well as symmetries, the new seven-point building block is constructed in terms of multiple polylogarithms. The results of this work are assembled in a computer-readable file attached to this submission (see supplementary material).

Overview. Section 2 briefly summarizes the systematics of planar scattering amplitudes in the multi-Regge limit in a self-contained way. Section 3 highlights the simplifications and restrictions implied by specializing to certain kinematic regions, or by passing from functions to symbols. In section 4, the two-loop analysis of the multi-Regge limit remainder 
function is revisited from the Regge cut point of view. Section 5 extends the analysis to three loops, where the remainder function can be decomposed into a few basic building blocks. The latter are discussed in section 6 . We construct a function for the seven-point building block in section 7 , and section 8 presents the conclusion.

Note added. The simultaneous paper [33] has some overlap with the present work. In particular, there is a connection between the "factorization theorem" of [33] and the application of the reduction identities (4.6) to the expansion of the $n$-point cut contribution carried out in this work.

\section{Background}

Multi-Regge kinematics. The $2 \rightarrow(n-2)$ multi-Regge limit is the $n$-particle generalization of the simple $s \gg t$ Regge limit for $2 \rightarrow 2$ scattering. To describe a general amplitude, we will use the $(3 n-10)$ independent Lorentz invariants

$$
\begin{aligned}
t_{j} & \equiv \boldsymbol{q}_{j}^{2}, \quad \boldsymbol{q}_{j} \equiv \boldsymbol{p}_{2}+\boldsymbol{p}_{3}+\cdots+\boldsymbol{p}_{j-1}, & & j=4, \ldots, n, \\
s_{j} & \equiv s_{j-1, j} \equiv\left(\boldsymbol{p}_{j-1}+\boldsymbol{p}_{j}\right)^{2}, & & j=4, \ldots, n, \\
\eta_{j} & \equiv \frac{s_{j} s_{j+1}}{\left(\boldsymbol{p}_{j-1}+\boldsymbol{p}_{j}+\boldsymbol{p}_{j+1}\right)^{2}}, & & j=4, \ldots, n-1 .
\end{aligned}
$$

Here, $\boldsymbol{p}_{1}, \ldots, \boldsymbol{p}_{n}$ are the $n$ external momenta. By convention, they are all incoming, but may have either energy sign. The $2 \rightarrow(n-2)$ multi-Regge limit is characterized by a large separation of rapidities among the produced particles. In terms of the above kinematic variables, the limit is attained for

$$
|s| \gg\left|s_{4}\right|, \ldots,\left|s_{n}\right| \gg t_{4}, \ldots, t_{n},
$$

where $s=\left(\boldsymbol{p}_{1}+\boldsymbol{p}_{2}\right)^{2}$ is the total energy. See figure 1 for an illustration of the kinematics. Many quantities in the multi-Regge limit only depend on the kinematics in the transverse space to the $\left(\boldsymbol{p}_{1}, \boldsymbol{p}_{2}\right)$ plane. We hence define

$$
\boldsymbol{p}_{j}=\alpha_{j} \boldsymbol{p}_{1}+\beta_{j} \boldsymbol{p}_{2}+\boldsymbol{p}_{j}^{\perp}, \quad \boldsymbol{p}_{1} \cdot \boldsymbol{p}_{j}^{\perp}=\boldsymbol{p}_{2} \cdot \boldsymbol{p}_{j}^{\perp}=0, \quad j=4, \ldots, n-1,
$$

and similarly for $\boldsymbol{q}_{4}, \ldots, \boldsymbol{q}_{n}$. It is often convenient to switch to complex variables $p_{j}, q_{j}$ whose real and imaginary parts equal the two components of the transverse momenta $\boldsymbol{p}_{j}^{\perp}$ and $\boldsymbol{q}_{j}^{\perp}$, respectively:

$$
\boldsymbol{p}_{j}^{\perp}=\left(\Re\left(p_{j}\right), \Im\left(p_{j}\right)\right), \quad \boldsymbol{q}_{j}^{\perp}=\left(\Re\left(q_{j}\right), \Im\left(q_{j}\right)\right) .
$$

Frequently used combinations of the transverse momenta are the complex anharmonic ratios

$$
w_{j}=\frac{p_{j-1} q_{j+1}}{q_{j-1} p_{j}}, \quad j=5, \ldots, n-1 .
$$

Planar $\mathcal{N}=4$ super Yang-Mills theory enjoys dual conformal invariance. Invariant quantities in this theory can thus only depend on conformally invariant cross ratios

$$
U_{i j} \equiv \frac{x_{i+1, j}^{2} x_{i, j+1}^{2}}{x_{i j}^{2} x_{i+1, j+1}^{2}}, \quad 3 \leq|i-j| \leq n-2
$$




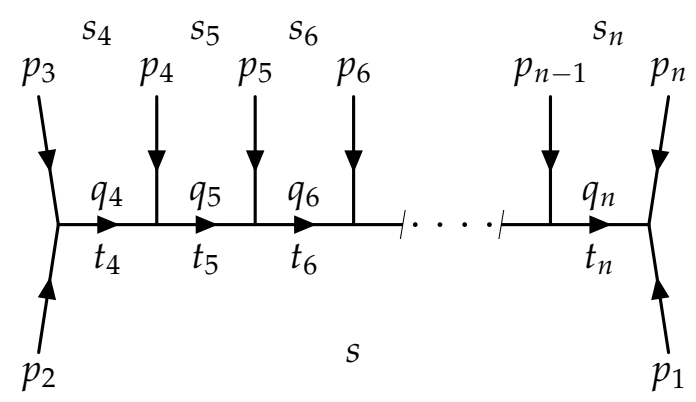

Figure 1. Kinematic variables.

of the dual coordinates

$$
\boldsymbol{p}_{j} \equiv x_{j}-x_{j-1}, \quad x_{i j}=x_{i}-x_{j} .
$$

A basis of kinematically independent invariant cross ratios is provided by

$$
u_{j, 1}=U_{j-3, j}, \quad u_{j, 2}=U_{j-2, n}, \quad u_{j, 3}=U_{1, j-1}, \quad j=5, \ldots, n-1 .
$$

In the multi-Regge limit, these cross ratios converge to 1 or 0 :

$$
u_{j, 1} \rightarrow 1, \quad u_{j, 2} \rightarrow 0, \quad u_{j, 3} \rightarrow 0 .
$$

The ratios of subleading terms remain finite, and are related to the anharmonic ratios $(2.7)$ via

$$
\frac{u_{j, 2}}{1-u_{j, 1}} \rightarrow \frac{1}{\left|1+w_{j}\right|^{2}}, \quad \frac{u_{j, 3}}{1-u_{j, 1}} \rightarrow \frac{\left|w_{j}\right|^{2}}{\left|1+w_{j}\right|^{2}}
$$

Kinematic regions. In order to understand the full analytic structure of the multi-Regge limit amplitude, it is important to analyze it in all physical kinematic regions. Our starting point will be the physical region in which the energies of all particles $3, \ldots, n$ are negative (which means that those particles are effectively outgoing, instead of incoming). In this region, all subenergies $s_{j}, j=4, \ldots, n$, are negative. ${ }^{1}$ In all other physical regions that we will consider, some of the particles $4, \ldots, n-1$ have positive energies (those particles become incoming), and hence some of the invariants $s_{j}$ become positive. These other regions are sometimes called "Mandelstam regions". ${ }^{2}$ They can be reached from the alloutgoing region by analytic continuation of the kinematics. The various regions will be labeled by the subsets $I \subset\{4, \ldots, n-1\}$ of particles whose energies have been continued to positive values. Alternatively, we will often label regions by $\rho=\left(\rho_{4}, \ldots, \rho_{n-1}\right) \in \mathbb{Z}_{2}^{n-4}$, with $\rho_{j}= \pm 1$ (or just $\rho_{j}= \pm$ ) indicating whether the respective particle has been flipped (its energy has been continued) $(-)$ or not $(+)$.

Importantly, the various regions become disconnected in the strict multi-Regge limit. That is to say, in order to continue the kinematics from one region to the other, one has to complexify the subenergies $s_{k}$ (e.g. by continuing them along big circles).

\footnotetext{
${ }^{1}$ The Minkowski metric is assumed to have signature $(-+++)$.

${ }^{2}$ One could also consider regions in which the energies of particles 3 and/or $n$ are positive, but those regions do not add further analytic structure to the amplitude, and will thus not be considered in the following.
} 
Multi-Regge limit amplitudes. Scattering in the multi-Regge limit is dominated by the exchange of "Reggeized gluons" (or "Reggeons"), which are effective particles that resum the contributions of entire classes of gluonic Feynman diagrams of all loop orders. The simplest example is the four-point amplitude in planar $\mathcal{N}=4$ super Yang-Mills theory, for which all perturbative contributions can be resummed and factorized into a single diagram:

$$
\mathcal{A}_{4}^{\mathrm{MRL}}=\overbrace{2}^{3} \overbrace{1}^{4}=\Gamma(t) s^{\omega(t)} \Gamma(t) .
$$

Here, $\bullet=\Gamma(t)$ is the gluon-gluon-Reggeon vertex (see e.g. [9]), and — stands for the exchange of a single Reggeon with propagator $s^{\omega(t)}$, where $\omega(t)$ is the (real-valued) Regge trajectory. At five points, two different kinematic regions can be considered: the produced particle 4 can either be flipped $(-)$ or not $(+)$. Strikingly, the factorization property of the four-point amplitude extends to this case: in both regions, the planar five-point amplitude again factorizes into a single diagram,

$$
\mathcal{A}_{5}^{\mathrm{MRL}( \pm)}={ }_{2}^{3} \overbrace{1}^{4} \overbrace{}^{5}=\Gamma\left(t_{4}\right) s_{4}^{\omega\left(t_{4}\right)} \Gamma_{45} s_{5}^{\omega\left(t_{5}\right)} \Gamma\left(t_{5}\right),
$$

where the (complex) gluon production vertex [11]

$$
\stackrel{j^{j}}{\oplus}=\Gamma_{j, j+1}=\left|\Gamma_{j, j+1}\right| e^{ \pm i \pi \hat{\omega}_{j, j+1}}, \quad \hat{\omega}_{j, j+1}=\hat{\omega}\left(t_{j}, t_{j+1}, \eta_{j}\right),
$$

only depends on the kinematic region through the sign of its phase.

A general $n$-point multi-Regge-limit amplitude in any given kinematic region $\rho$ receives contributions from Regge pole [34] as well as Mandelstam cut terms [35, 36],

$$
\mathcal{A}_{n, \rho}^{\mathrm{MRL}}=\mathcal{A}_{n, \rho}^{\text {Regge pole }}+\mathcal{A}_{n, \rho}^{\text {Mandelstam cut }} .
$$

Both the pole terms and the cut terms depend on the kinematic region $\rho$. The origin of the Mandelstam cut terms are non-trivial contributions from multi-Reggeon bound state exchange in intermediate $t$-channels. For planar amplitudes of up to five points, such contributions are suppressed by powers of $1 / N_{\mathrm{c}}$, and the amplitudes factorize as indicated above. At six points, the first cut term appears, in the region $(--)$ where both intermediate momenta have been flipped [10]. In a generic region $\rho$, the six-point amplitude therefore reads [11, 27]

$$
\mathcal{A}_{6, \rho}^{\mathrm{MRL}}=(\text { pole terms })^{\rho}+c_{6,1,4}^{\rho}{ }_{2}^{3}
$$

where the region-dependent coefficient $c_{6,1,4}^{\rho}$ is non-vanishing for $\rho=(--)$. Here, the cut diagram stands for all contributions from two-Reggeon bound state exchange in the $t_{5}$ channel. This picture generalizes to higher multiplicities: the planar $n$-point multi-Regge 
limit amplitude is a sum of region-dependent Regge pole terms as well as Mandelstam cut contributions with region-dependent coefficients [9-11, 26-28, 37, 38]:

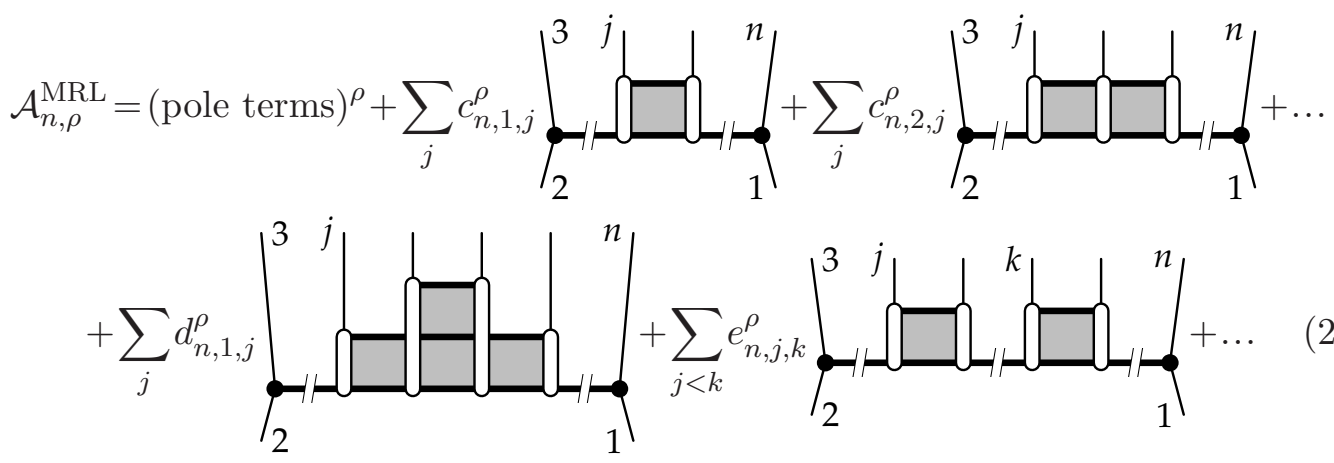

Here, the symbol - stands for the insertion of zero or more complex gluon production vertices (2.15). For planar amplitudes, the number of exchanged Reggeons can at most increase or decrease by one when passing from one $t$-channel to the next. ${ }^{3}$ All other contributions are suppressed by powers of $1 / N_{\mathrm{c}}$. The pole terms as well as the cut-term prefactors can in principle be obtained from the general quantum field theory principles of locality \& unitarity. The procedure particularly relies on an expansion of the amplitude into a sum of terms that each have no overlapping energy discontinuities, following the Steinmann relations [40]. Determining the cut contributions in this way is a very intricate and tedious procedure that has to be carried out region by region. This formidable task has been completed for the seven-point amplitude [28], and a study of the eight-point case is underway [38], but a generalization to higher multiplicities appears difficult. Below, we will see that the coefficients $c_{n, b, j}$ are actually fixed by the two-loop analysis [31].

In fact, the Mandelstam criterion [36] significantly constrains the set of cut terms that can contribute to any given kinematic region: it asserts that any cut contribution in which the multi-Reggeon states span the adjacent $t$-channels $t_{j}, \ldots, t_{k}$ cannot contribute to regions in which $s_{j-1}>0$ or $s_{k+1}>0$, that is ${ }^{4}$

$$
c_{n, k-j, j}^{\rho}=0 \quad \text { if } \quad \rho_{j-1}=\rho_{j} \quad \text { or } \quad \rho_{k}=\rho_{k+1},
$$

and similarly for the further coefficients in (2.18). Here, the subscripts $n, b$, and $j$ in $c_{n, b, j}^{\rho}$ label the total number of particles, the number of $t$-channels taking part in the multi-Reggeon state, and the produced gluon that bounds the multi-Reggeon state on the left. For example, as indicated above, the six-particle cut term (2.17) is only present in the (--) region:

$$
c_{6,1,4}^{(++)}=c_{6,1,4}^{(+-)}=c_{6,1,4}^{(-+)}=0 .
$$

BDS and remainder function. The MHV amplitudes of planar $\mathcal{N}=4$ super YangMills theory can be decomposed into two factors:

$$
\mathcal{A}_{n}^{\mathrm{MHV}}=\mathcal{A}_{n}^{\mathrm{BDS}} R_{n}
$$

\footnotetext{
${ }^{3}$ The number $M_{n}$ of admissible diagrams that can contribute to the $n$-point amplitude, as a sequence in $n$, equals the Motzkin sequence, OEIS A001006 [39], with $M_{n} / M_{n-1} \rightarrow 3$ for $n \rightarrow \infty$.

${ }^{4}$ The reason is that in such cases, one of the Feynman loop integrals can be closed trivially, since all singularities lie on the same side of the integration contour [41].
} 
Here, $\mathcal{A}_{n}^{\mathrm{BDS}}$ is the Bern-Dixon-Smirnov amplitude [7], wich equals the tree-level amplitude times the exponentiated one-loop amplitude, and which in fact produces the correct all-loop four-point and five-point amplitudes. Starting at six points, it however fails to reproduce the correct Regge pole contributions, and it misses all Regge cut terms (beyond one loop) [9-11]. Hence it cannot be the full amplitude, but has to be corrected by a non-trivial remainder function $R_{n}$. Since the BDS amplitude correctly captures all infrared singularities and dual conformal weights, the remainder function is infrared finite and dual conformally invariant, and thus can only depend on dual conformally invariant cross ratios (2.8). By definition, it is only non-trivial starting from six points and two loops.

Passing to the multi-Regge limit, and stripping off the universal absolute value, the BDS amplitude reduces to a region-dependent phase factor. From the latter, one can separate off a conformally invariant, infrared finite $\operatorname{part} \exp \left(i \delta_{n}^{\rho}\right)$, which again is regiondependent, and contains the finite part of the one-loop Regge cut terms [11, 27]

$$
\frac{\mathcal{A}_{n}^{\mathrm{BDS}, \mathrm{MRL}, \rho}}{\Gamma\left(t_{4}\right)\left|s_{4}^{\omega_{4}}\right|\left|\Gamma_{45}\right|\left|s_{5}^{\omega_{5}}\right|\left|\Gamma_{56}\right|\left|s_{6}^{\omega_{6}}\right| \ldots\left|s_{n-1}^{\omega_{n-1}}\right|\left|\Gamma_{n-1, n}\right|\left|s_{n}^{\omega_{n}}\right| \Gamma\left(t_{n}\right)}=\exp \left(i \phi_{n}^{\rho}\right) \exp \left(i \delta_{n}^{\rho}\right) .
$$

The universal denominator is a generalization of the five-point amplitude (2.14), and it subsumes all dependence on the absolute values of the gluon production vertices $\Gamma_{k, k+1}$ and Reggeon propagators $s_{k}^{\omega_{k}}$. The region-dependent phase $\exp \left(i \phi_{n}^{\rho}\right)$ absorbs the remaining infrared divergences. The finite, conformally invariant piece $\exp \left(i \delta_{n}^{\rho}\right)$ combines in a nontrivial way with the remainder function to a region-dependent linear combination of reduced pole and cut terms $[27,28]$ :

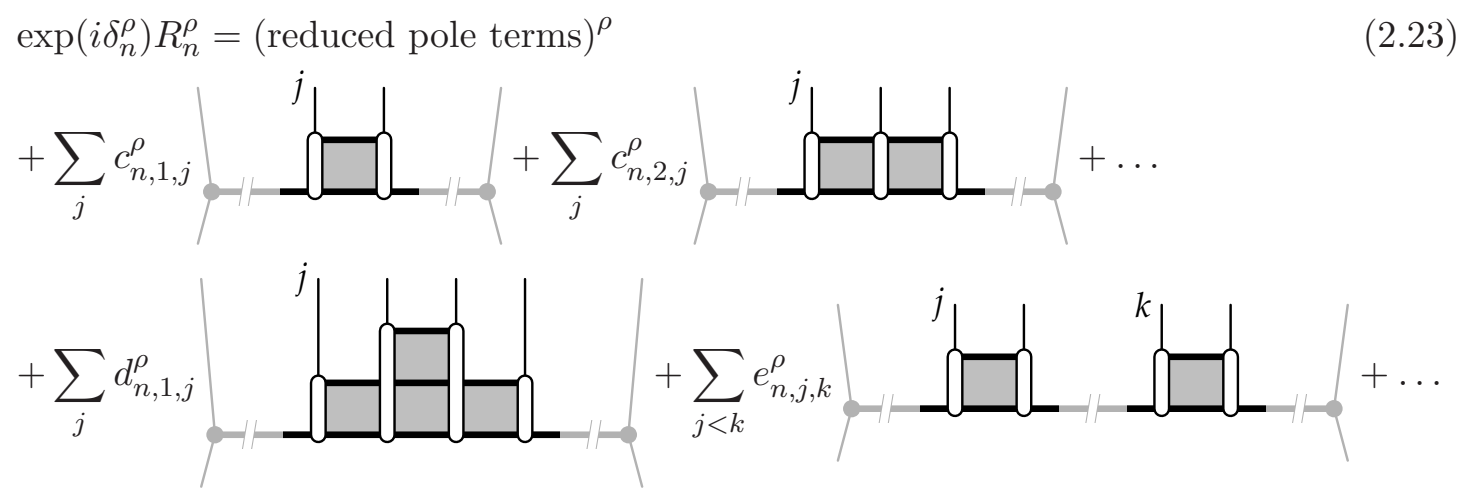

Here, the grayed-out parts of the cut diagrams have been divided out, and the (black) cut pieces stand for the remainder after the division.

Factorized cut integrals. All reduced cut terms in (2.23) are infrared-finite, conformally invariant functions of the complex anharmonic ratios $w_{k}(2.7)$. Just like the pole terms of the four-point and five-point amplitudes, they enjoy the virtue of Regge factorization, in the following sense: the multi-Reggeon bound states that propagate in the intermediate $t$-channels are governed by the BFKL $[1-3]$ and BKP $[4,5]$ equations. The solutions to these equations are most naturally expressed in terms of their $\mathrm{SL}(2, \mathbb{C})$ representation labels $(n, \nu)$. Expressing all quantities in terms of these variables, the cut contribution factorizes into a simple product: reading a cut diagram from left to right, each 
$t$-channel $m$-Reggeon state contributes one BFKL (or BKP) Green's function $G_{m}\left(n_{k}, \nu_{k}\right)$, each gluon emission that increments or decrements the number of exchanged reggeons from $m$ to $m \pm 1$ contributes an impact factor $\Phi_{m, m \pm 1}\left(n_{k-1}, \nu_{k-1}, n_{k}, \nu_{k}\right)$, and each intermediate gluon $k$ that gets emitted from an $m$-Reggeon bound state contributes a central emission block $C_{m}\left(n_{k-1}, \nu_{k-1}, n_{k}, \nu_{k}\right)$. Obtaining the full cut contribution requires completing the state sums in all $t$-channels by summing and integrating over all $n_{k}$ and $\nu_{k}$. The summation and integration amounts to a Fourier-Mellin transform from the $\left(n_{k}, \nu_{k}\right)$ variables to the complex anharmonic ratios $w_{k}$ that provide the kinematic dependence.

The subsequent analysis will focus on the cuts of the type shown in the middle line of (2.23). For those terms, only the simplest impact factors [10]

$$
\begin{aligned}
\Phi_{\mathrm{L}, k} & \equiv \Phi_{0,1}\left(n_{k}, \nu_{k}\right)=\frac{1}{2} \frac{(-1)^{n}}{i \nu_{k}+n_{k} / 2}\left(\frac{q_{k-1}}{p_{k-1}}\right)^{-i \nu_{k}-n_{k} / 2}\left(\frac{\bar{q}_{k-1}}{\bar{p}_{k-1}}\right)^{-i \nu_{k}+n_{k} / 2}+\mathcal{O}(g), \\
\Phi_{\mathrm{R}, k} \equiv \Phi_{1,0}\left(n_{k}, \nu_{k}\right) & =-\frac{1}{2} \frac{1}{i \nu_{k}-n_{k} / 2}\left(\frac{q_{k+1}}{p_{k}}\right)^{i \nu_{k}+n_{k} / 2}\left(\frac{\bar{q}_{k+1}}{\bar{p}_{k}}\right)^{i \nu_{k}-n_{k} / 2}+\mathcal{O}(g)
\end{aligned}
$$

and emission blocks [26]

$$
\begin{aligned}
C_{k} \equiv & C_{1}\left(n_{k}, \nu_{k}, n_{k+1}, \nu_{k+1}\right)=-\frac{1}{2}\left(\frac{q_{k+1}}{p_{k}}\right)^{i \nu_{k}+n_{k} / 2}\left(\frac{\bar{q}_{k+1}}{\bar{p}_{k}}\right)^{i \nu_{k}-n_{k} / 2} \cdot \\
& \cdot\left(\frac{q_{k}}{p_{k}}\right)^{-i \nu_{k+1}-n_{k+1} / 2}\left(\frac{\bar{q}_{k}}{\bar{p}_{k}}\right)^{-i \nu_{k+1}+n_{k+1} / 2} \tilde{C}\left(n_{k}, \nu_{k}, n_{k+1}, \nu_{k+1}\right)+\mathcal{O}(g)
\end{aligned}
$$

are needed. The required Green's function stems from the BFKL color-octet channel and takes the form [10]

$$
G_{k} \equiv G_{2}\left(n_{k}, \nu_{k}\right)=\varepsilon_{k}^{g E_{n_{k}, \nu_{k}}}
$$

Here, $\varepsilon_{k} \equiv-\sqrt{u_{k, 2} u_{k, 3}}$ are combinations of "small" cross ratios (2.10) that approach zero in the multi-Regge limit, $E_{n, \nu}$ is the BFKL color-octet eigenvalue, and

$$
g \equiv \frac{g_{\mathrm{YM}}^{2} N_{\mathrm{c}}}{8 \pi^{2}}
$$

is the planar coupling constant. The general two-Reggeon cut term $f_{k}$ spanning $k t$-channels therefore takes the form $[26,30]^{5}$

$$
\begin{aligned}
& f_{k}\left(\varepsilon_{5}, \ldots, \varepsilon_{k+4} ; w_{5}, \ldots, w_{k+4}\right) \equiv \sum_{n_{5}, \ldots, n_{k+4}}^{4} \int \mathrm{d} \nu_{5} \ldots \mathrm{d} \nu_{k+4} \Phi_{\mathrm{L}, 5} \varepsilon_{5}^{g E_{n_{5}, \nu_{5}}} C_{5} \varepsilon_{6}^{g E_{n_{6}, \nu_{6}}} C_{6} \ldots C_{k+3} \varepsilon_{k+4}^{g+4}= \\
& \quad=i g \varepsilon_{k_{n_{k+4}, \nu_{k+4}}}^{k+4} \Phi_{\mathrm{R}, k+4} .
\end{aligned}
$$

One can see that the exponentials of kinematic variables in the impact factors and emission blocks indeed combine into Fourier-Mellin integral transformation kernels

$$
w_{k}^{i \nu_{k}+n_{k} / 2} \bar{w}_{k}^{i \nu_{k}-n_{k} / 2}=\rho_{k}^{2 i \nu_{k}} e^{i n_{k} \varphi_{k}} \quad \text { for } \quad w_{k}=\rho_{k} e^{i \varphi_{k}} .
$$

\footnotetext{
${ }^{5}$ The cut contribution is normalized such that the cut coefficient $c_{6,1,4}^{\rho=(-)}$ of the six-point remainder function becomes unity. This choice differs from the normalization used in [28] by a factor of $2 i$.
} 
Perturbative expansion. The expression (2.28) is valid to all orders in the coupling $g$, where all coupling dependence is contained in the impact factors $\Phi_{\mathrm{L}, \mathrm{R}}$, the emission blocks $C_{k}$, and the BFKL eigenvalues $E_{n_{k}, \nu_{k}}$. Upon a perturbative expansion, the BFKL Green's functions (2.26) expand in powers of $g$ and of $\log \left(\varepsilon_{k}\right)$; the latter are the large logarithms that are characteristic of the multi-Regge limit. Including subleading terms of the BFKL eigenvalues, impact factors, and emission blocks, the cut contribution (2.28) at each order $g^{\ell}$ in the coupling constant becomes a polynomial of degree $(\ell-1)$ in the large $\operatorname{logarithms} \log \varepsilon_{k}$. Retaining only the leading terms in large logarithms amounts to the leading logarithmic approximation (LLA), the first subleading terms constitute the next-to-leading logarithmic approximation (NLLA), and so on. At order $g^{\ell}$, there are LLA terms of order $\log \left(\varepsilon_{k}\right)^{\ell-1}$ all the way to $\mathrm{N}^{\ell-1}$ LLA terms of order $\log \left(\varepsilon_{k}\right)^{0}$. At a given loop order, the coefficient of each monomial in $\log \left(\varepsilon_{k}\right)$ is a function of the kinematics that exclusively depends on the complex anharmonic ratios $w_{k}(2.7)$.

\section{Symbols and regions}

Transcendentality and symbols. Scattering amplitudes in planar $\mathcal{N}=4$ super YangMills theory display the property of uniform (or maximal) transcendentality, which means that every term in the $\ell$-loop amplitude has the same transcendentality (or transcendental weight) $2 \ell$. This concept relies on the assumption that the amplitude can be expanded in products of multiple polylogarithms (iterated integrals over dlog integrands, MPLs for short) [14], $\pi$, and zeta values. ${ }^{6}$ Every $m$-fold iterated integral is assigned transcendentality $m$. Zeta values can be defined as MPLs evaluated on certain values, and they inherit the transcendentality of their parent functions. For example, the polylogarithms $\operatorname{Li}_{m}(x)$ as well as the zeta values $\zeta_{m}$ have transcendentality $m$, and $\pi$ has transcendental weight 1 . Under multiplication, transcendentality behaves additively.

Multiple polylogarithms obey many functional identities, which makes them unwieldy, especially in expressions with many terms. All such functional relations trivialize when one projects all MPLs to their symbols $[45] .{ }^{7}$ The latter discard all information contained in the choice of integration base point. In particular, the symbols are agnostic of all ambiguities lying in the choice of functional branch. Since all branch ambiguities of MPLs have subleading functional transcendentality (transcendentality of functional origin, as opposed to numerical transcendentality), one typically discards all terms of subleading functional transcendentality when mapping an expression to its symbol.

When projecting the amplitude to its symbol, the expression (2.23) simplifies considerably: the reduced pole terms consist of trigonometric functions whose arguments include factors of $\pi[27,28]$, hence their perturbative expansion contains extra powers of $\pi$, which implies that they carry subleading functional transcendental weight; they therefore get

\footnotetext{
${ }^{6}$ It is expected that this class of functions is not sufficient to describe all amplitudes to all orders in general kinematics. For example, elliptic integrals appear in the ten-point $\mathrm{N}^{3} \mathrm{MHV}$ amplitude [42]. However, based on the singularity structure of the integrand, it is safe to assume that all MHV amplitudes can be expressed as rational polynomials of multiple polylogarithms and zeta values [43, 44], and multi-Regge limit MHV amplitudes inherit this property.

${ }^{7}$ For reviews, see $[46,47]$.
} 
discarded. Cut terms that involve more than two Reggeons stem from double (or higher) discontinuities, hence they also have subleading transcendentality and get projected out. Terms with multiple disconnected multi-Reggeon states (such as the last term in (2.23)) are products of lower-loop cut terms, hence also these have subleading transcendental weight and get discarded. On the left hand side of the equation, the factor $\exp \left(i \delta_{n}^{\rho}\right)$ can be truncated to 1 , since all higher terms again include additional factors of $\pi$. In summary, at the level of the symbol, the remainder function is a linear combination of two-Reggeon cut terms:

$$
\left.R_{n}^{\rho} \simeq \sum_{j} c_{n, 1, j}^{\rho}\right|_{-} ^{j}+\left.\sum_{j} c_{n, 2, j}^{\rho}\right|_{-} ^{j}+\ldots
$$

Here, " $\simeq$ " denotes equality at the symbol level. ${ }^{8}$ Moreover, here and in the following, the remaining (black) cut pieces are understood to be one-loop subtracted, as the one-loop part is (by definition) contained in the BDS factor that has been divided out. The dots stand for further two-Reggeon cut terms that span any number of adjacent emitted gluons.

Symbols and regions. At the symbol level, the discontinuity of an iterated integral along a closed continuation path only depends on the overall winding numbers of the path around the singular points of the integrand. From this property alone, it follows [31] that the symbols $\mathrm{S}[\cdot]$ of the multi-Regge-limit remainder function in the various kinematic regions obey the relations

$$
\mathrm{S}\left[R_{n}^{I}\right]=\sum_{\{k, l\} \subset I} \mathrm{~S}\left[R_{n}^{\{k, l\}}\right]
$$

and

$$
\mathrm{S}\left[R_{n}^{\{k, l\}}\right]=\mathrm{S}\left[R_{n}^{[k, l]}\right]-\mathrm{S}\left[R_{n}^{[k, l-1]}\right]-\mathrm{S}\left[R_{n}^{[k+1, l]}\right]+\mathrm{S}\left[R_{n}^{[k+1, l-1]}\right] .
$$

These relations hold independently of the loop order. The first relation states that the symbol in any region $I \subset\{4, \ldots, n-1\}$ is a sum of symbols in regions $\{k, l\}$ where only two momenta $\boldsymbol{p}_{k}$ and $\boldsymbol{p}_{l}$ are flipped. The second relation in turn expresses the symbol in those two-flip regions as a linear combination of symbols in regions where all flipped momenta $k, \ldots, l$ are adjacent, labeled by $[k, l]$. It is therefore sufficient to consider the symbol in those all-adjacent regions.

Note that, since the cut terms can be assumed to be functionally independent, the relations (3.2), (3.3) among symbols imply identical relations for the cut prefactors $c_{n, b, j}^{\rho}$ in the various regions:

$$
c_{n, b, j}^{I}=\sum_{\{k, l\} \subset I} c_{n, b, j}^{\{k, l\}}, \quad c_{n, b, j}^{\{k, l\}}=c_{n, b, j}^{[k, l]}-c_{n, b, j}^{[k, l-1]}-c_{n, b, j}^{[k+1, l]}+c_{n, b, j}^{[k+1, l-1]} .
$$

It is not difficult to see that these relations are consistent with the Mandelstam criterion (2.19) described above. They completely determine the coefficients of all two-Reggeon

\footnotetext{
${ }^{8}$ Strictly speaking, the symbol vanishes, since the right-hand side contains an overall factor of $2 \pi i$. What is meant by " $\simeq$ " is that the symbols on both sides agree after pulling out the overall $2 \pi i$ factor.
} 
cut contributions of the type shown in (3.1) to the $n$-point remainder function in any kinematic region $\rho$ in terms of the coefficients $c_{n, b, j}^{[k, l]}$ of these cut terms in the all-adjacent regions $\rho=[k, l]$.

In fact, the Mandelstam criterion (2.19) implies that there is only a single two-Reggeon cut contribution to the $n$-point multi-Regge limit remainder function in any all-adjacent region $[k, l]$, namely

$$
R_{n, \text { cut }}^{[k, l]}=c_{n, l-k, k}^{[k, l]}
$$

where the subscript "cut" indicates that the Regge pole terms are not included, and the dots stand for the omission of $(l-k-2)$ emission blocks. In other words,

$$
c_{n, b, j}^{[k, l]}=0 \quad \text { unless } \quad j=k, \quad \text { and } \quad b=l-k .
$$

In particular, the cut terms in all such regions equal (up to variable substitution and the prefactors) the cut terms of the $(l-k+5)$-point remainder function in the region where all intermediate momenta are flipped:

$$
R_{n, \mathrm{cut}}^{[k, l]}\left(\varepsilon_{k+1}, \ldots, \varepsilon_{l} ; w_{k+1}, \ldots, w_{l}\right)=\frac{c_{n, l-k, k}^{[k, l]}}{c_{n^{\prime}, n^{\prime}-4,4}^{\left[4, n^{\prime}-1\right]}} R_{n^{\prime}, \text { cut }}^{\left[4, n^{\prime}-1\right]}\left(\varepsilon_{k+1}, \ldots, \varepsilon_{l} ; w_{k+1}, \ldots, w_{l}\right),
$$

with $n^{\prime}=l-k+5$. Since the symbol of the remainder funtion is agnostic of the pole terms, the equations (3.5) and (3.7) hold for the full remainder function at the symbol level.

\section{Two-loop expansion}

We now want to analyze the two-Reggeon contribution (3.5) for any number of gluons at the perturbative level. The following deconstruction is not restricted to symbols, but holds at the level of full functions. By definition, all cut diagrams of the type (3.5) are understood to be one-loop subtracted. The simplest case involves only two emitted gluons. Perturbatively expanding the BFKL Green's function and the impact factors, this simplest diagram consists of three terms at the two-loop level:

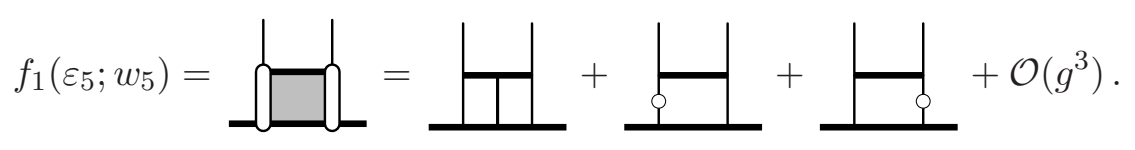

Here, a naked line for the impact factor stands for its leading contribution (2.24), whereas additional dots denote loop corrections. A vertical line in the $t$-channel two-Reggeon state stands for the one-loop (order $g^{1}$ ) piece of the BFKL Green's function (2.26),

$$
G_{2}\left(n_{k}, \nu_{k}\right)=\varepsilon_{k}^{g E_{n_{k}, \nu_{k}}}=1+g E_{n_{k}, \nu_{k}}^{(0)} \log \left(\varepsilon_{k}\right)+\mathcal{O}\left(g^{2}\right),
$$

where

$$
E_{n_{k}, \nu_{k}}=\sum_{\ell=0}^{\infty} g^{\ell} E_{n_{k}, \nu_{k}}^{(\ell)}
$$


is the expansion of the BFKL eigenvalue. Due to the factor $\log \left(\varepsilon_{k}\right)$ in the one-loop Green's function, the first term in (4.1) provides the leading logarithmic approximation (LLA) at this two-loop order. The subleading NLLA contribution consists of the second and third diagrams, which have no line insertions, and stem from the trivial piece $G_{2}(g=0)=1$ of the Green's function.

Turning to the longer two-Reggeon cut that appears in the (---) region of the sevenpoint remainder function, the two-loop expansion yields five terms,

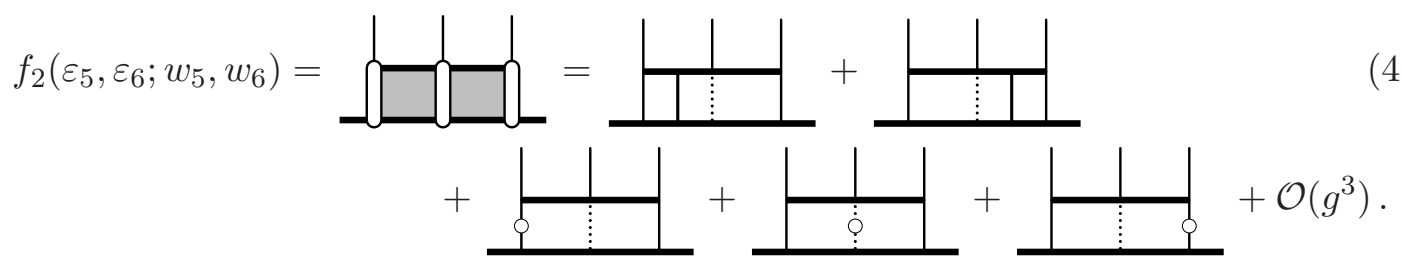

Here, the emission block makes its first appearance. A plain dotted line stands for the leading-order emission block (2.25), and additional dots again denote loop corrections. The LLA piece now consists of two terms, where either of the Green's functions in the first or second $t$-channel have been expanded to one-loop order. Hence the first term is proportional to $\log \left(\varepsilon_{5}\right)$, while the second term is proportional to $\log \left(\varepsilon_{6}\right) \cdot{ }^{9}$ The second line provides the three NLLA terms.

A key fact for the subsequent analysis is the following observation [26]: any number of adjacent leading-order emission blocks, not separated by BFKL eigenvalue insertions, can be absorbed in a neighboring leading-order impact factor (again not separated by BFKL eigenvalue insertions). The result is the original impact factor, whose momentum gets replaced by the sum of combined momenta. Similarly, any number of adjacent leadingorder emission blocks can be combined into a multi-gluon emission block, whose functional form is identical to the single-gluon block, but whose outgoing momentum is replaced by the sum of all combined momenta. Diagrammatically, we will denote these identities as

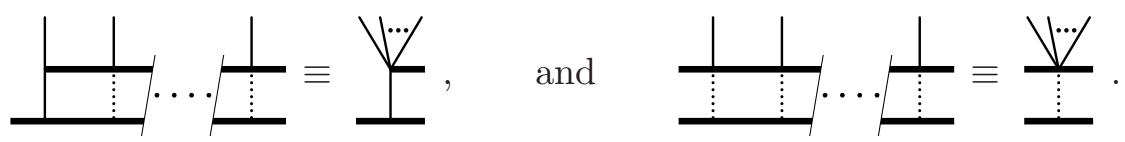

Here, the dots stand for the insertion of any number of leading-order emission blocks. The identity for impact factors (left) was demonstrated in [26], and the identity for emission blocks (right) follows straightforwardly. For completeness, the identities are derived in appendix A. Using these identities, one can reduce almost all diagrams in (4.4) to six-point diagrams. For example,
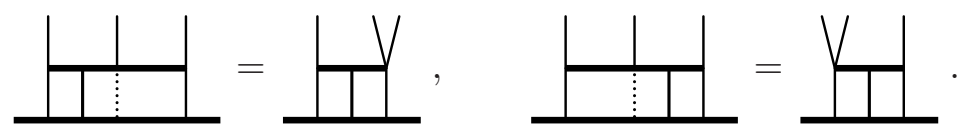

Each term in the two-loop expression (4.4) a priori depends on both complex anharmonic ratios $w_{5}$ and $w_{6}(2.7)$. But due to the identity (4.6), it is clear that all dependence of the

\footnotetext{
${ }^{9}$ Without loss of generality, it is assumed that the leftmost particle at the beginning of the cut is particle 4.
} 
first term in (4.4) on $w_{5}$ and $w_{6}$ factors into a dependence on the single complex ratio

$$
v_{5,6 ; 5} \equiv \frac{p_{4} q_{7}}{q_{4}\left(p_{5}+p_{6}\right)}=\frac{w_{5}}{\left(1+\frac{1}{w_{6}}\right)} .
$$

Similarly, the second term in (4.4) only depends on the single complex ratio

$$
v_{5,6 ; 6} \equiv \frac{\left(p_{4}+p_{5}\right) q_{7}}{q_{4} p_{6}}=\left(1+w_{5}\right) w_{6} .
$$

Restricting to the LLA (the first line in (4.4)), and using the identities (4.6), the threeparticle cut therefore reduces to a sum of two copies of the two-particle cut,

$$
f_{2,(2)}^{\mathrm{LLA}}\left(\varepsilon_{5}, \varepsilon_{6} ; w_{5}, w_{6}\right)=f_{1,(2)}^{\mathrm{LLA}}\left(\varepsilon_{5} ; v_{5,6 ; 5}\right)+f_{1,(2)}^{\mathrm{LLA}}\left(\varepsilon_{6} ; v_{5,6 ; 6}\right) .
$$

Promoting this equation to the full two-loop cut contribution (including the NLLA piece) requires adding an extra NLLA term to the equation:

$$
f_{2,(2)}\left(\varepsilon_{5}, \varepsilon_{6} ; w_{5}, w_{6}\right)=f_{1,(2)}\left(\varepsilon_{5} ; v_{5,6 ; 5}\right)+f_{1,(2)}\left(\varepsilon_{6} ; v_{5,6 ; 6}\right)+g_{2}\left(v_{5,6 ; 5}, v_{5,6 ; 6}\right),
$$

where

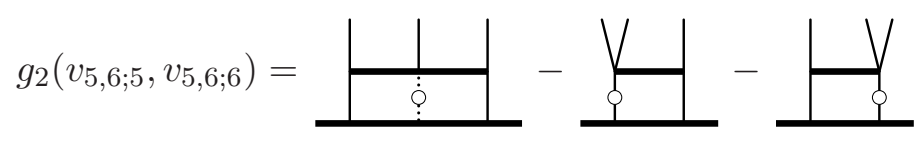

is a finite function of $v_{5,6 ; 5}$ and $v_{5,6 ; 6}$ (or of $w_{4}$ and $w_{5}$ via the relations (4.7), (4.8)). Here, the last two terms appear in the two $f_{1,(2)}$ terms but not in $f_{2,(2)}$ and thus need to be subtracted. They are, by analogy with (4.5), defined by evaluating the one-loop impact factors on the sums of momenta $p_{4}+p_{5}$ and $p_{5}+p_{6}$, respectively.

This two-loop analysis straightforwardly generalizes to the cut contribution for any number of particles. Using the identities (4.5), the LLA part of the general cut at two loops can be written as

$$
f_{k,(2)}^{\mathrm{LLA}}\left(\varepsilon_{5}, \ldots, \varepsilon_{4+k} ; w_{5}, \ldots, w_{4+k}\right)=\sum_{j=5}^{4+k} \bigvee^{4} \bigvee^{j} \bigvee^{4+k}=\sum_{j=5}^{4+k} f_{1,(2)}^{\mathrm{LLA}}\left(\varepsilon_{j} ; v_{5,4+k ; j}\right) .
$$

Here, the variables

$$
v_{k, l ; j} \equiv \frac{q_{k-1}-q_{j}}{q_{k-1}} \frac{q_{l+1}}{q_{j}-q_{l+1}}=\frac{\left(1+\left(1+\left(\ldots\left(1+w_{k}\right) w_{k+1}\right) \ldots\right) w_{j-1}\right) w_{j}}{1+\left(1+\left(\ldots\left(1+\frac{1}{w_{l}}\right) \frac{1}{w_{l-1}}\right) \ldots\right) \frac{1}{w_{j+1}}}
$$

for $j=k, \ldots, l$ are anharmonic ratios that generalize (4.7), (4.8); they are obtained by grouping the adjacent momenta $p_{k-1}+\cdots+p_{j-1}=q_{k-1}-q_{j}$ and $p_{j}+\cdots+p_{l}=q_{j}-q_{l+1}$. The inversion of this formula is

$$
w_{j}=\frac{\left(v_{k, l ; j-1}-v_{k, l ; j}\right)\left(1+v_{k, l, j+1}\right)}{\left(1+v_{k, l ; j-1}\right)\left(v_{k, l ; j}-v_{k, l ; j+1}\right)},
$$

assuming the boundary conditions $v_{k, l ; k-1}=0$ and $v_{k, l ; l+1}=\infty$. Including the NLLA terms of $f_{k,(2)}$ and $f_{1,(2)}$ on both sides of equation (4.12), and again applying the reduction 
identities (4.5), one can see that all subleading terms combine into a sum of seven-point NLLA pieces $g_{2}$ (4.11), evaluated with different complex ratios:

$$
f_{k,(2)}\left(\varepsilon_{5}, \ldots, \varepsilon_{4+k} ; w_{5}, \ldots, w_{4+k}\right)=\sum_{j=5}^{4+k} f_{1,(2)}\left(\varepsilon_{j} ; v_{j}\right)+\sum_{j=5}^{3+k} g_{2}\left(v_{j}, v_{j+1}\right), \quad v_{j} \equiv v_{5,4+k ; j}
$$

This concludes the two-loop analysis of the general two-Reggeon cut (3.5). For any number of emitted particles, the latter can be deconstructed into a sum of two building blocks, one of them being the simplest two-particle cut $f_{1}$, the other being the NLLA remainder $g_{2}$ of the three-particle cut $f_{2}$.

Using equation (3.5), the result (4.15) directly implies an analogous relation for the cut piece of the two-loop remainder function in the region $(--\ldots-)$ where all momenta have been flipped,

$$
R_{n,(2), \text { cut }}^{\mathrm{MRL},(--\ldots-)}\left(\varepsilon_{5}, \ldots, \varepsilon_{n-1} ; w_{5}, \ldots, w_{n-1}\right)=\frac{c_{n}}{c_{6}} \sum_{j=5}^{n-1} R_{6,(2), \text { cut }}^{\mathrm{MRL},(--)}\left(\varepsilon_{j} ; v_{j}\right)+c_{n} \sum_{j=5}^{n-2} g_{2}\left(v_{j}, v_{j+1}\right),
$$

with the abbreviations $c_{n} \equiv c_{n, n-5,4}^{[4, n-1]}$ and $v_{j} \equiv v_{5, n-1 ; j}$. With the help of (3.7), very similar relations hold for the remainder function symbol in any region $[k, l]$ where any number of adjacent momenta have been flipped.

Relation to previous work. At leading logarithmic order, the relation (4.15) together with the variable map (4.13) has been obtained before [26]. Here, we have generalized it to the full two-loop level, including the NLLA terms. In fact, an explicit study [31] of the known two-loop symbol [32] has lead to the slightly stronger observation

$$
R_{n,(2), \text { cut }}^{\mathrm{MRL},(-\ldots-)}\left(\varepsilon_{5}, \ldots, \varepsilon_{n-1} ; w_{5}, \ldots, w_{n-1}\right) \simeq \sum_{j=5}^{n-1} R_{6,(2), \text { cut }}^{\mathrm{MRL},(--)}\left(\varepsilon_{j} ; v_{j}\right)+c_{7} \sum_{j=5}^{n-2} g_{2}\left(v_{j}, v_{j+1}\right)
$$

Also this result had been obtained previously at leading logarithmic order [48]. Comparing (4.16) with (4.17), one finds that the coefficients of all simple two-Reggeon cut contributions must be identical, ${ }^{10}$

$$
c_{n, l-k, k}^{[k, l]}=c_{6,1,4}^{[4,5]}=1, \quad n \geq 7, \quad 4 \leq k<l<n .
$$

Here, the second equality follows from the deliberate choice of normalization (2.28) for the cut integral.

\footnotetext{
${ }^{10}$ Since [31] analyzed the two-loop symbol for up to ten points, the equality has only been rigorously established for $n \leq 10$.
} 


\section{Three-loop expansion}

We are now in a position to extend the previous analysis to the three-loop order. At three loops, the simplest cut contribution $f_{1}$ expands to

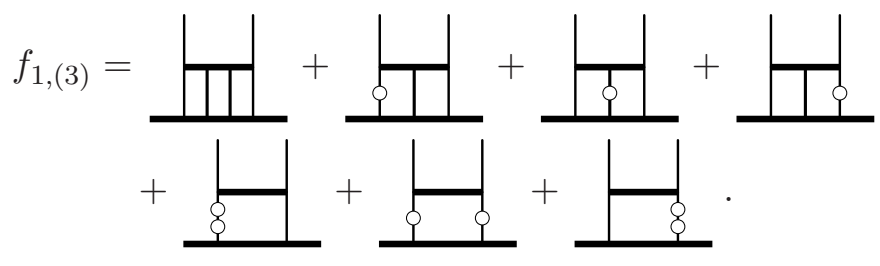

Compared to the two-loop case, there are a few new ingredients at three loops: two line insertions in the two-Reggeon state (as in the first term) stand for terms where the BFKL Green's function (2.26) has been expanded to second order in the coupling $g$, while the BFKL eigenvalue $E_{n, \nu}$ has been kept at leading order. A line insertion dressed with a dot stands for one power of the one-loop correction to the eigenvalue $E_{n, \nu}$. Each line (leading order or loop corrected) comes with one power of the respective large logarithm $\log \left(\varepsilon_{k}\right)$. In other words, expanding

$$
\begin{aligned}
G_{2}\left(n_{k}, \nu_{k}\right) & =\varepsilon_{k}^{g E_{n_{k}, \nu_{k}}}= \\
& =1+g E_{n_{k}, \nu_{k}}^{(0)} \log \left(\varepsilon_{k}\right)+\frac{1}{2} g^{2}\left(E_{n_{k}, \nu_{k}}^{(0)} \log \left(\varepsilon_{k}\right)\right)^{2}+g^{2} E_{n_{k}, \nu_{k}}^{(1)} \log \left(\varepsilon_{k}\right)+\mathcal{O}\left(g^{3}\right)
\end{aligned}
$$

where $E_{n_{k}, \nu_{k}}^{(\ell)}$ is the $\ell$-loop BFKL eigenvalue, the third term in (5.2) produces the first term in (5.1), whereas the fourth term in (5.2) produces the third term in (5.1). The first term in (5.1) constitutes the LLA part, the next three terms provide the NLLA contribution, and the three terms on the second line form the NNLLA piece.

Passing now to the longer cut $f_{2}$, one finds the following terms at three loops and leading logarithmic order:

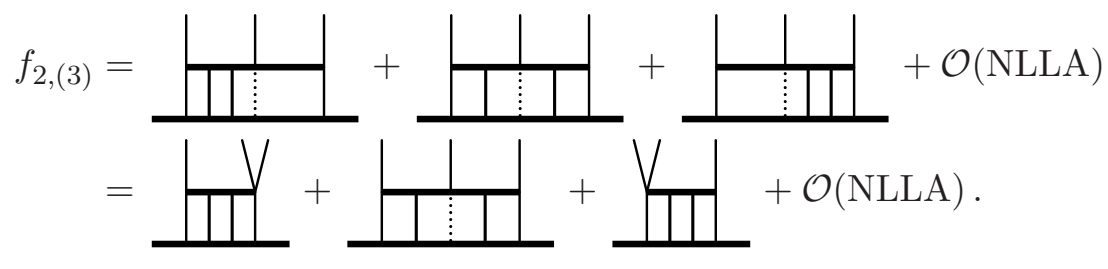

As shown in the second line, two of the LLA diagrams can again be reduced to six-point diagrams, using (4.5). But, unlike in the two-loop case, one LLA diagram remains that cannot be reproduced by six-point data. Removing the six-point pieces by subtracting two instances of $f_{1,(3)}$ functions (5.1), one finds the remainder (without loss of generality, the emitted gluons are labeled by $\{4,5,6\}$ )

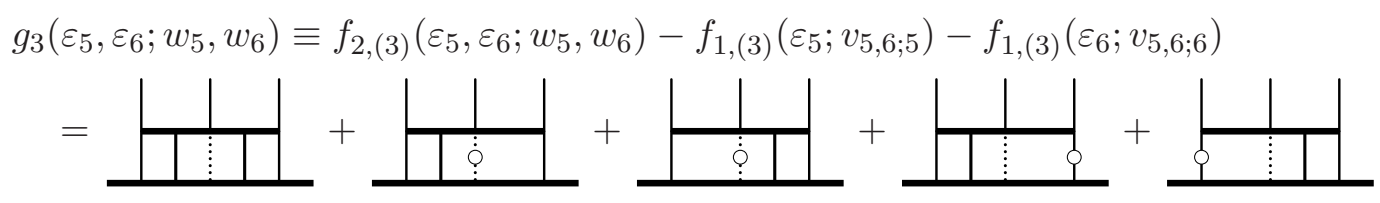




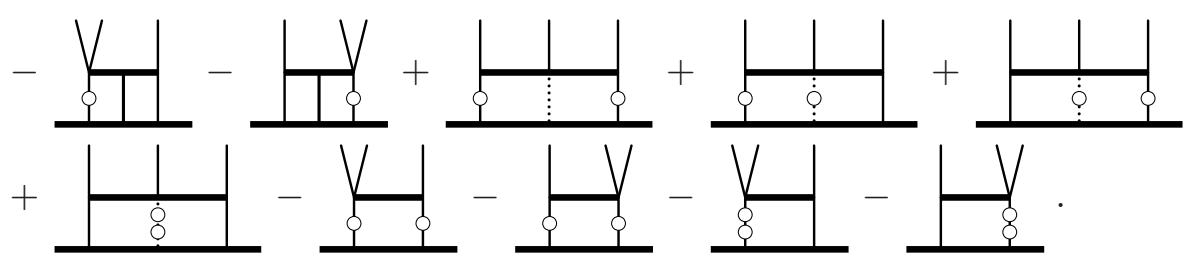

Note that all terms involving the next-to-leading-order BFKL eigenvalue are captured by the short cut terms $f_{1(3)}$. It is now straightforward to see that the general $k$-point cut diagram $f_{k,(3)}$, to leading logarithmic order, becomes a sum of six-point functions $f_{1,(3)}$ and seven-point functions $g_{3}:^{11}$

$$
\begin{aligned}
& f_{k,(3)}^{\mathrm{LLA}}\left(\varepsilon_{5}, \ldots, \varepsilon_{4+k} ; w_{5}, \ldots, w_{4+k}\right) \\
&=\sum_{j=5}^{4+k} f_{1,(3)}^{\mathrm{LLA}}\left(\varepsilon_{j} ; v_{5,4+k ; j}\right)+\sum_{i=5}^{3+k} \sum_{j=i+1}^{4+k} g_{3}^{\mathrm{LLA}}\left(\varepsilon_{i}, \varepsilon_{j} ; v_{5, j-1 ; i}, v_{i+1,4+k ; j}\right) .
\end{aligned}
$$

Including all NLLA and NNLLA diagrams in the functions $f_{k,(3)}, f_{1,(3)}$, and $g_{3}$ on both sides of the above equation, and judiciously organizing all terms, one finds that the subleading contributions can be combined into two further NLLA building blocks $g_{\mathrm{L}}, g_{\mathrm{R}}$, and one further NNLLA building block $h$. The full three-loop cut function $f_{k,(3)}$ can be written as

$$
\begin{aligned}
& f_{k,(3)}\left(\varepsilon_{5}, \ldots, \varepsilon_{k+4} ; w_{5}, \ldots, w_{k+4}\right)=\sum_{j=5}^{k+4} f_{1,(3)}\left(\varepsilon_{j} ; v_{5,4+k ; j}\right)+\sum_{i=5}^{k+3} \sum_{j=i+1}^{k+4} g_{3}\left(\varepsilon_{i}, \varepsilon_{j} ; v_{5, j-1 ; i}, v_{i+1, k+4 ; j}\right) \\
& +\sum_{i=5}^{k+2} \sum_{j=i+1}^{k+3} g_{\mathrm{L}}\left(\varepsilon_{i} ; v_{5, j-1 ; i}, v_{i+1, j ; j}, v_{j+1, k+4 ; j+1}\right)+\sum_{i=5}^{k+2} \sum_{j=i+2}^{k+4} g_{\mathrm{R}}\left(\varepsilon_{j} ; v_{5, i ; i}, v_{i+1, j-1 ; i+1}, v_{i+2, k+4 ; j}\right) \\
& +\sum_{i=5}^{k+1} \sum_{j=i+2}^{k+3} h\left(v_{5, i ; i}, v_{i+1, j-1 ; i+1}, v_{i+2, j ; j}, v_{j+1, k+4 ; j+1}\right) .
\end{aligned}
$$

The NLLA building block $g_{\mathrm{L}}$ depends on four intermediate momenta. It takes the form

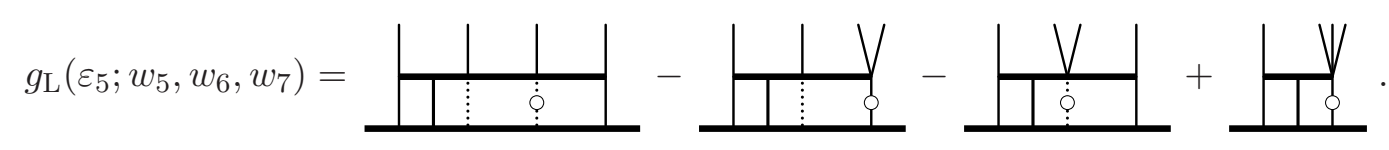

In the third term of (5.6), this function gets summed over partitions of the sequence of momenta $\left(p_{4}, \ldots, p_{k+4}\right)$ into subsequences

$$
\left(p_{4}, \ldots, p_{i-1}\right), \quad\left(p_{i}, \ldots, p_{j-1}\right), \quad\left(p_{j}\right), \quad \text { and } \quad\left(p_{j+1}, \ldots, p_{k+4}\right) .
$$

The building block $g_{\mathrm{R}}$ is a mirror of $g_{\mathrm{L}}$ :

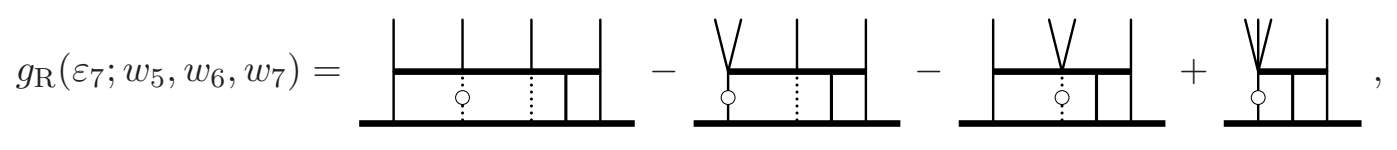

\footnotetext{
${ }^{11}$ Note that, contrary to the two-loop case (4.11), the three-loop building block $g_{3}$ is defined in terms of the original cross ratios $w_{5}, w_{6}$ rather than the combinations $v_{5,6 ; 5}, v_{5,6 ; 6}$.
} 
and in the fourth term of (5.6), it gets summed over the partitions

$$
\left(p_{4}, \ldots, p_{i-1}\right), \quad\left(p_{i}\right), \quad\left(p_{i+1}, \ldots, p_{j-1}\right), \quad \text { and }\left(p_{j}, \ldots, p_{k+4}\right) .
$$

Finally, the $\mathrm{N}^{2} \mathrm{LLA}$ building block $h$ reads:

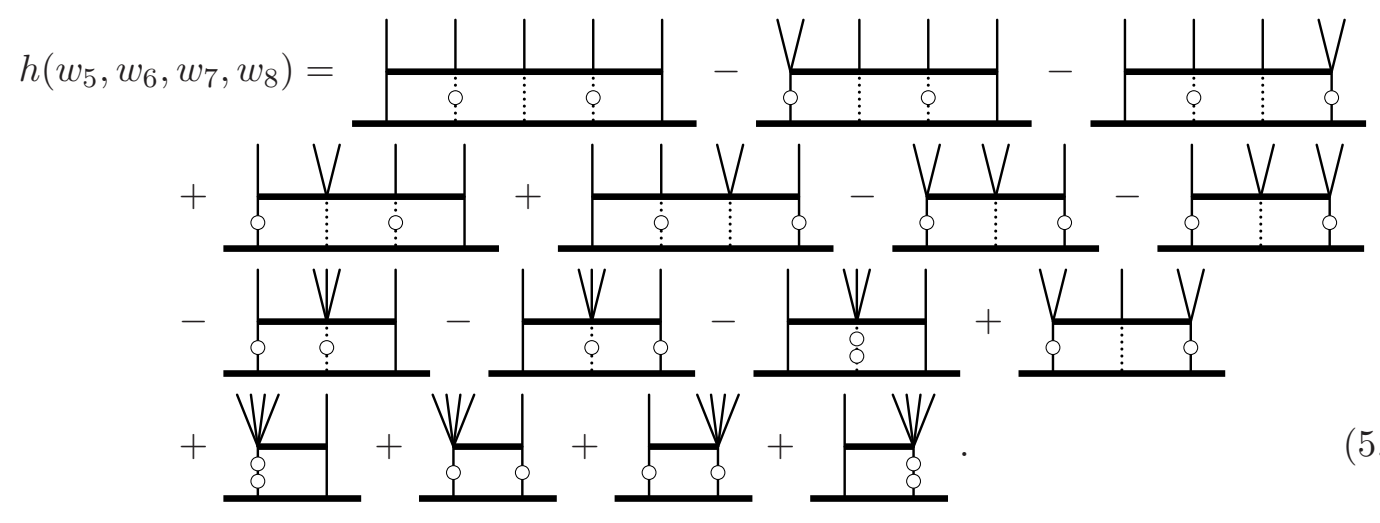

The last term in (5.6) sums this function over partitions of the intermediate momenta into subsequences

$$
\left(p_{4}, \ldots, p_{i-1}\right), \quad\left(p_{i}\right), \quad\left(p_{i+1}, \ldots, p_{j-1}\right), \quad\left(p_{j}\right), \quad \text { and } \quad\left(p_{j+1}, \ldots, p_{k+4}\right) .
$$

For the case $k=3$, which is relevant for the eight-point remainder function in the $(----)$ region, the last sum in (5.6) has to be replaced by the single term $\tilde{h}\left(w_{5}, w_{6}, w_{7}\right)$, where $\tilde{h}$ is obtained from $h$ (5.11) by removing the middle particle (and the associated LO emission block, if applicable).

Using (3.5), the deconstruction (5.6) implies an analogous relation for the three-loop remainder function in the region $\rho=[4, n-1]=(--\ldots-)$ where all intermediate momenta have been flipped:

$$
\begin{aligned}
& R_{n,(3), \text { cut }}^{(--\ldots-)}\left(\varepsilon_{4}, \ldots, \varepsilon_{n-2} ; w_{4}, \ldots, w_{n-2}\right)=\sum_{j=5}^{n-1} R_{6,(3), \text { cut }}^{(--)}\left(\varepsilon_{j} ; v_{j}\right)+\sum_{\substack{i, j=5 \\
i<j}}^{n-1} g_{3}\left(\varepsilon_{i}, \varepsilon_{j} ; v_{5, j-1 ; i}, v_{i+1, n-1 ; j}\right) \\
& +\sum_{i=5}^{n-3} \sum_{j=i+1}^{n-2} g_{\mathrm{L}}\left(\varepsilon_{i} ; v_{5, j-1 ; i}, v_{i+1, j ; j}, v_{j+1, n-1 ; j+1}\right)+\sum_{i=5}^{n-3} \sum_{j=i+2}^{n-1} g_{\mathrm{R}}\left(\varepsilon_{j} ; v_{5, i ; i}, v_{i+1, j-1 ; i+1}, v_{i+2, n-1 ; j}\right) \\
& +\sum_{i=5}^{n-4} \sum_{j=i+2}^{n-2} h\left(v_{5, i ;}, v_{i+1, j-1 ; i+1}, v_{i+2, j ; j}, v_{j+1, k+4 ; j+1}\right) .
\end{aligned}
$$

Here, the identities (4.18) among the cut coefficients have already been taken into account. Via (3.7), equivalent relations hold for the remainder function in all regions $\rho=[k, l]$ where any number of adjacent momenta $\{k, \ldots, l\}$ has been flipped. In more general regions, the remainder function receives contributions from further cut terms (of the type shown in the last line of (2.23)). Passing to the remainder function symbol, these further cut terms drop out (due to their subleading functional transcendentality). Thus, by (3.2), (3.3), the deconstruction (5.13) implies a decomposition of the remainder function symbol in any kinematic region in terms of the symbols of the five building blocks $f_{1,(3)}, g_{3}, g_{L}, g_{R}$, and $h$. 


\section{Building blocks}

In principle, each term in the perturbative expansion of the Regge cut diagram (3.5) can be computed from the integral representation (2.28), once the expressions for the BFKL eigenvalue, impact factor, and central emission block are known to the desired perturbative order. In the previous sections, we have shown that, by judiciously organizing all terms in the expansion, the $n$-point two-loop and three-loop cut contributions can be reconstructed from a few basic building blocks that are functions of the anharmonic ratios $w_{j}$. Once these building block functions are known, the Regge cut contribution to the remainder function can be computed via the formulas (4.16), (5.13).

Here, we will content ourselves with treating the building block functions at the level of the symbol. The symbol of the two-loop NLLA building block $g_{2}\left(v_{1}, v_{2}\right)$ has been obtained [31] by taking the multi-Regge limit of the known two-loop remainder function symbol [32] and using the decomposition (4.17).

At three loops, both the six-point and seven-point remainder function symbols are known $[24,49]$. By its definition, this data is sufficient to extract the symbol of the building block $g_{3}$ (5.4), which contains LLA, NLLA, and NNLLA parts. Applying in turn the first line of the three-loop decomposition (5.13), this admits a reconstruction of the $n$-point remainder function symbol at leading logarithmic order.

We compute the multi-Regge limit symbol of the three-loop remainder function in the same way as for the two-loop symbol. The procedure is detailed in [31], here we only give a brief summary: starting with the known six-point and seven-point symbols for general kinematics, we expand all first entries in terms of the cross ratios (2.10) via the symbol rule

$$
(x y) \otimes(z)=(x) \otimes(z)+(y) \otimes(z) .
$$

Next, we collect all terms with the same cross ratio $U_{k, l}$ in the first entry, strip off the first entry, and multiply by $2 \pi i$. The result is the symbol of the discontinuity under continuation along the path $U_{k, l} \rightarrow e^{2 \pi i} U_{k, l}$. In order to obtain the multi-Regge limit symbol of each discontinuity, we express the kinematic invariants in the symbol entries in terms of the OPE variables

$$
\left\{T_{j}, S_{j}, F_{j}\right\}=\left\{e^{-\tau_{j}}, e^{\sigma_{j}}, e^{i \phi_{j}}\right\}, \quad j=5, \ldots, n-1,
$$

of [18], ${ }^{12}$ set $S_{j}=1 /\left(T_{j} r_{j}\right)$, and take the limit $T_{j} \rightarrow 0$, keeping only the leading term in each entry. For the six-point case,

$$
r_{5}^{2}=w_{5} \bar{w}_{5}, \quad F_{5}^{2}=w_{5} / \bar{w}_{5}, \quad T_{5}^{2}=\varepsilon_{5} / r_{5},
$$

whereas for seven points,

$$
\begin{array}{lll}
r_{5}^{2}=w_{6} \bar{w}_{6}, & F_{5}^{2}=w_{6} / \bar{w}_{6}, & T_{5}^{2}=\varepsilon_{6} / r_{5}, \\
r_{6}^{2}=1 / w_{5} \bar{w}_{5}, & F_{6}^{2}=\bar{w}_{5} / w_{5}, & T_{6}^{2}=\varepsilon_{5} / r_{6} .
\end{array}
$$

\footnotetext{
${ }^{12}$ Compared to [18], we cyclically shift the momentum twistors, such that $Z_{i}^{\text {here }}=Z_{i+1}^{\text {BSV }}$.
} 
Finally, again expanding all terms via (6.1), one can extract all large logarithms via the shuffle relations

$$
\begin{aligned}
& \log \left(\varepsilon_{j}\right)(x \otimes y \otimes \cdots \otimes z)=\left(\varepsilon_{j} \otimes x \otimes y \otimes \cdots \otimes z\right)+\left(x \otimes \varepsilon_{j} \otimes y \otimes \cdots \otimes z\right) \\
& \quad+\left(x \otimes y \otimes \varepsilon_{j} \otimes \cdots \otimes z\right)+\cdots+\left(x \otimes y \otimes \cdots \otimes \varepsilon_{j} \otimes z\right)+\left(x \otimes y \otimes \cdots \otimes z \otimes \varepsilon_{j}\right) .
\end{aligned}
$$

At seven points and three loops, the resulting expression for each discontinuity is a degreetwo polynomial in $\log \left(\varepsilon_{5}\right)$ and $\log \left(\varepsilon_{6}\right)$, whose coefficients are symbols with five entries that exclusively depend on $w_{5}, w_{6}$, and their complex conjugates. Starting in the kinematic region $(+++)$ in which no intermediate momentum is flipped, each other kinematic region is associated with specific winding numbers for all cross ratios $U_{k, l}$. Summing the corresponding discontinuities then yields the remainder function symbol in the respective kinematic region. In particular, the region (---) that contains the three-particle cut $f_{2}$, only the cross-ratio $U_{2,6}(2.8)$ winds non-trivially. Applying the change of variables (4.7), (4.8) and subtracting the respective six-point three-loop symbols (5.4), one finally obtains the symbol of the building block $g_{3}$.

The NLLA and NNLLA building blocks (5.7), (5.9), (5.11) first appear in the three-loop eight-point and nine-point amplitudes, and can thus not (yet) be extracted from available perturbative data. In principle these functions could be computed term by term from the integral representation (2.28). While the BFKL eigenvalue and impact factor are known explicitly to $\mathrm{N}^{2}$ LLA and $\mathrm{N}^{3}$ LLA $[10,12,20,37,50]$, and relating the multi-Regge limit to the Wilson loop OPE [51] led to all-order proposals [52], the missing ingredient is the NLO and NNLO central emission block (2.25).

In principle, the NLO emission block could be extracted from the building block $g_{2}$ by subtracting the two reducible terms and inverting the Fourier-Mellin transform. This however requires knowledge of the full function $g_{2}$, which at present is only known at leading transcendental weight [31].

The attached MATHEMATICA file MRL3LLA .m (see supplementary material) contains the symbols for the building blocks $R_{6,(3)}^{\mathrm{MRL}}$ and $g_{3}$, as well as a function that reconstructs the three-loop leading-logarithmic-order remainder function symbol in any kinematic region from these building blocks.

Note on the alphabet. The three-loop three-particle building block $g_{3}\left(\varepsilon_{5}, \varepsilon_{6} ; w_{5}, w_{6}\right)$ has the same alphabet $\aleph$ (letters appearing in the entries of the symbol) as the two-loop three-particle building block $g_{2}\left(w_{5}, w_{6}\right)$ :

$$
\aleph_{w}=\left\{w_{5}, 1+w_{5}, w_{6}, 1+w_{6}, 1+w_{6}+w_{5} w_{6}\right\} \cup\{\text { c.c. }\},
$$

where "c.c." stands for the complex conjugate set of letters. Using the expansion (5.13), and expanding all variables $v_{k, l ; j}$ in terms of $w_{5}, \cdots, w_{n-1}$, the alphabet (of the terms in the first line) becomes big and complicated for larger $n$. Had one started with the $n$ point symbol, it would have been difficult to guess the variable transformation (4.13) that simplifies the alphabet and symbol terms.

Beyond seven points, the full alphabet of the remainder function remains unknown, even in the multi-Regge limit. At six and seven points, the alphabet apparently does 
not change with the loop order, with the full alphabet already visible at two loops. It appears likely that this pattern breaks at eight points (beyond the leading logarithmic approximation), since this is the first instance at which the three-loop building blocks involve more independent legs than the two-loop building blocks. It would be interesting to work out the consequences of the deconstruction (5.13) on the higher-point alphabets in more detail.

\section{The function $g_{3}$}

Clearly, it is desirable to obtain the building blocks of the multi-Regge limit amplitude at function level. The function for the three-loop six-point building block $f_{1,(3)}$ has been derived in [49]. Here, we focus on constructing the function for the new three-loop seven-point building block $g_{3}$, which, together with $f_{1,(3)}$, determines the three-particle cut $f_{2,(3)}$ (5.6). While we will not be able to determine the function $g_{3}$ completely, we can severely constrain it using the knowledge of its symbol as well as further constraints from symmetry and consistency with the collinear limit.

Structure of the function $\boldsymbol{g}_{\mathbf{3}}$. MHV amplitudes in multi-Regge kinematics are rational polynomials in multiple polylogarithms, $\pi$, and (multiple) zeta values, where all occuring monomials have the same (uniform) transcendental weight [53]. At loop order $\ell$, the remainder function has weight $2 \ell$. The cut terms that make up the remainder function in the multi-Regge limit are discontinuities of the full remainder function and thus carry an overall factor $2 \pi i$, which is therefore multiplied by a function of uniform weight $(2 \ell-1)$. Collecting large logarithms, the function $g_{3}$ decomposes as follows: ${ }^{13}$

$$
g_{3}\left(\varepsilon_{5}, \varepsilon_{6} ; x, y\right)=2 \pi i \sum_{m, n=0}^{1} \log \left(\varepsilon_{5}\right)^{i} \log \left(\varepsilon_{6}\right)^{j}\left(g_{3}^{m, n}(x, y)+2 \pi i h_{3}^{m, n}(x, y)\right) .
$$

At each order in the large $\operatorname{logarithms} \log \left(\varepsilon_{j}\right)$, we have split the function into real parts $g_{3}^{m, n}$ and imaginary parts $h_{3}^{m, n}$, each of which is a fixed-weight combination of multiple polylogarithms and zeta values with real coefficients.

Ordinary multiple polylogarithms. In order to construct the function $g_{3}$ by matching a general ansatz to its symbol, we first need an irreducible basis of multiple polylogarithms of the right class. Multiple polylogarithms, also called Goncharov polylogarithms [14], can be defined recursively as iterated integrals

$$
G\left(a_{1}, \ldots, a_{n} ; z\right) \equiv \begin{cases}\frac{1}{n !} \log ^{n} z & \text { if } a_{1}=\ldots=a_{n}=0, \\ \int_{0}^{z} \frac{d t}{t-a_{1}} G\left(a_{2}, \ldots, a_{n} ; t\right) & \text { otherwise }\end{cases}
$$

with $G(; z)=1$. The sequence of parameters $\left(a_{1}, \ldots, a_{n}\right)$ is called the weight vector, and the length of the weight vector equals the transcendental weight (or transcendentality)

\footnotetext{
${ }^{13}$ We call the function $g_{3}\left(\varepsilon_{5}, \varepsilon_{6} ; x, y\right) \equiv g_{3}\left(\varepsilon_{5}, \varepsilon_{6} ; w_{5}(x, y), w_{6}(x, y)\right)$ by the same name as $g_{3}\left(\varepsilon_{5}, \varepsilon_{6} ; w_{5}, w_{6}\right)$.
} 
of the function $G\left(a_{1}, \ldots, a_{n} ; z\right)$. Multiple zeta values are defined in terms of multiple polylogarithms evaluated at unity, and inherit their transcendental weight: $\zeta_{k}$ has weight $k, \zeta_{j, k}$ has weight $j+k$, and so forth. $\pi$ has weight 1 .

As noted in [31], using the variables

$$
x=-v_{6}=-\left(1+w_{5}\right) w_{6}, \quad y=-1 / v_{5}=-\frac{1+w_{6}}{w_{5} w_{6}},
$$

the alphabet (6.6) of the symbol of $g_{3}$ becomes

$$
\aleph_{x y}=\{x, x-1, y, y-1, x y-1\} \cup\{\text { c.c. }\} .
$$

Multiple polylogarithms whose symbols draw their entries from this alphabet belong to the class of two-dimensional harmonic polylogarithms (2dHPLs) [54]. A generating set for these is given by ${ }^{14}$

$$
\left\{G(\vec{a}, x) \mid a_{i} \in\{0,1\}\right\} \cup\left\{G(\vec{a}, 1 / y) \mid a_{i} \in\{0,1, x\}\right\} \cup\{\text { c.c. }\},
$$

where $\{$ c.c. $\}$ stands for the complex conjugates of the previous sets. Multiple polylogarithms satisfy shuffle and stuffle algebra relations, hence the above generating set is overcomplete. An irreducible basis of generators is provided by the subset whose weight vectors form Lyndon words in the ordered sets of letters $\{0,1\}$ and $\{0,1, x\}$, respectively [55]. Including the complex conjugate generators, the resulting irreducible set consists of 10,8 , 20, 42, and 108 basis functions at weights $1,2,3,4$, and 5 . Including all possible products of lower-weight functions yields 10,63, 320, 1433, and 5190 linearly independent terms at weights $1,2,3,4$, and 5 .

Single-valuedness. Besides the consistency with its known symbol, the function $g_{3}$ has to satisfy various constraints. One of them is single-valuedness: due to unitarity, a physical amplitude can only have branch points where one of the cross ratios vanishes (or becomes infinite). Since the cross ratios are expressed in terms of absolute squares of the complex variables $w_{5}$ and $w_{6}(2.12)$, a rotation $\left(w_{5}-z, \bar{w}_{5}-\bar{z}\right) \rightarrow\left(e^{+2 \pi i}\left(w_{5}-z\right), e^{-2 \pi i}\left(\bar{w}_{5}-\bar{z}\right)\right)$ around any point $z$ in the complex plane can never let a cross ratio wind around zero (or infinity). The same is true for rotations of $w_{6}$, and therefore also for rotations of $x$ and $y$. The conclusion is that the coefficient functions (7.1) of $g_{3}$ must be single-valued functions of the complex variables $x$ and $y$. This property has been essential for the determination of the six-point multi-Regge limit to high loop orders [12, 19, 56, 57].

One could in principle implement the single-valuedness constraint by first constructing a function using the basis (7.5) and then requiring all monodromies to vanish. However, it turns out that the single-valuedness constraint can be satisfied directly at the level of the basis: a suitable basis of single-valued multiple polylogarithms was recently constructed for any number of points [33]. ${ }^{15}$ We can therefore satisfy the single-valuedness constraint by

\footnotetext{
${ }^{14}$ The choice of generating set is not unique. We used a different basis in [31], but found the choice (7.5) more suitable for the present analysis.

${ }^{15}$ See also [23].
} 
employing the single-valued basis, without losing generality. ${ }^{16}$ The single-valued basis can be constructed purely algebraically from the basis of ordinary multiple polylogarithms (7.5) using the Hopf algebra structure that underlies the multiple polylogarithm algebra [14]: each holomorphic element $G$ of the ordinary basis (7.5) gets promoted to a single-valued function $G^{\mathbf{s}}$ by the single-valued map

$$
s: G(\vec{a}, z) \mapsto G^{\mathbf{s}}(\vec{a}, z) \equiv(-1)^{|\vec{a}|} \mu(\bar{S} \otimes \mathrm{id}) \Delta G(\vec{a}, z),
$$

where $\Delta$ is the coproduct, id is the identity, $\bar{S}$ is the complex conjugate of the antipode map of the Hopf algebra, and $\mu$ denotes the multiplication operator $\mu(a \otimes b)=a \cdot b$. The details are spelled out in section 3.4.3 of [33], and we will not reproduce them here. The antiholomorphic elements of (7.5) can equally be promoted to single-valued functions, which however are not independent from the single-valued functions generated from the holomorphic elements. A full basis of single-valued 2dHPLs is therefore provided by the single-valued completions of the holomorphic elements of the ordinary basis (7.5). Since this halves the size of the algebra basis, it significantly reduces the number of linearly independent elements in a general ansatz at any fixed weight. For example, while a general (real) weight-five ansatz constructed from the ordinary basis (7.5) as well as zeta values has 6305 terms (and therefore as many undetermined coefficients), the corresponding ansatz constructed from the single-valued basis has only 756 terms.

To summarize, the single-valued algebra basis that we will employ is

$$
\left\{G^{\mathbf{s}}(\vec{a}, x) \mid \vec{a} \in \operatorname{Lyn}\{0,1\}\right\} \cup\left\{G^{\mathbf{s}}(\vec{a}, 1 / y) \mid \vec{a} \in \operatorname{Lyn}\{0,1, x\}\right\},
$$

where $\operatorname{Lyn}\{0,1\}$ and $\operatorname{Lyn}\{0,1, x\}$ denote the sets of Lyndon words formed from the ordered sets of letters $\{0,1\}$ and $\{0,1, x\}$, respectively. Every single-valued function $G^{\mathbf{s}}(\vec{a}, z)$ is constructed from the ordinary multiple polylog $G(\vec{a}, z)$ according to the algebraic prescription (7.6). In addition, we assume that $\left\{\zeta_{2}, \zeta_{3}, \zeta_{2,3}, \zeta_{5}\right\}$ form the algebraically independent set of (multiple) zeta values up to weight five.

The ansatz and symbol constraints. We start with a general polynomial in singlevalued basis functions (7.7) and zeta values, such that all monomials have identical total weight. Given that the three-loop amplitude in general kinematics has weight six, and taking into account the overall factor of $2 \pi i$ as well as the large $\operatorname{logarithms} \log \left(\varepsilon_{5}\right), \log \left(\varepsilon_{6}\right)$, one finds that the LLA real part $g_{3}^{1,1}$ has to have weight three, the NLLA real parts $g_{3}^{1,0}$ and $g_{3}^{0,1}$ have weight four, and the NNLLA real part $g_{3}^{0,0}$ has weight five. The corresponding imaginary parts have weight one less than the real parts, due to the extra factor $2 \pi i$. The sizes of the general ansätze for all component functions are displayed in table 1 . The symbol of the function $g_{3}$ uniquely fixes all terms in the real parts $g_{3}^{m, n}$ with the highest functional weight, that is all terms that are free of zeta values. We can perform the match

\footnotetext{
${ }^{16}$ In the first revision of this paper, I had constructed the function $g_{3}$ using the basis (7.5), which was the state of the art at the time the preprint of this paper appeared on the arXiv. I thank the JHEP referee for requesting a construction based on the single-valued basis that was published at around the same time [33], and which significantly reduces the number of free parameters that remain after applying all constraints.
} 


\begin{tabular}{|rrrrrrrrrr|}
\hline Function & $g_{3}^{1,1}$ & $h_{3}^{1,1}$ & $g_{3}^{1,0}$ & $h_{3}^{1,0}$ & $g_{3}^{0,1}$ & $h_{3}^{0,1}$ & $g_{3}^{0,0}$ & $h_{3}^{0,0}$ & total \\
\hline General ansatz & 71 & 20 & 236 & 71 & 236 & 71 & 756 & 236 & 1697 \\
Match to symbol & 6 & 20 & 25 & 71 & 25 & 71 & 91 & 236 & 545 \\
Parity invariance & 6 & 16 & 21 & 51 & 21 & 51 & 67 & 151 & 384 \\
Target-projectile symmetry & 4 & 10 & 21 & 51 & 0 & 0 & $?$ & 83 & 236 \\
Vanishing collinear limit I & 2 & 7 & 15 & 44 & 0 & 0 & 55 & 76 & 199 \\
Consistency with the WLOPE I & 1 & 5 & 11 & 37 & 0 & 0 & 49 & 71 & 174 \\
Vanishing collinear limit II & 1 & 5 & 11 & 37 & 0 & 0 & 45 & 65 & 164 \\
Consistency with the WLOPE II & 1 & 5 & 11 & 37 & 0 & 0 & 42 & 59 & 155 \\
\hline
\end{tabular}

Table 1. Numbers of free parameters in the components of the function $g_{3}$ before imposing constraints, after matching to the known symbols, and after imposing various constraints. The first two components constitute the LLA part, the next four functions represent the NLLA part, and the last two functions form the NNLLA part of the function $g_{3}$.

by expanding the single-valued functions $G^{\mathbf{s}}(\vec{a}, z)$ into combinations of ordinary multiple polylogarithms $G(\cdot, \cdot)$, and by applying the symbol map

$$
\begin{aligned}
\mathrm{S}\left[G\left(a_{1}, \ldots, a_{n} ; z\right)\right]=\sum_{i=1}^{n} & \left(\mathrm{~S}\left[G\left(a_{1}, \ldots, \hat{a}_{i}, \ldots, a_{n} ; z\right)\right] \otimes\left(a_{i}-a_{i-1}\right)\right. \\
& \left.-\mathrm{S}\left[G\left(a_{1}, \ldots, \hat{a}_{i}, \ldots, a_{n} ; z\right)\right] \otimes\left(a_{i}-a_{i+1}\right)\right),
\end{aligned}
$$

where $a_{0}=z, a_{n+1}=0$, and hatted indices are omitted. The match to the symbol fixes the majority of terms in the real parts $g_{3}^{m, n}$ (see table 1), but the symbol is insensitive to all terms of subleading functional weight, including all terms in the imaginary parts $h_{3}^{m, n}$.

Parity invariance and target-projectile symmetry. While the terms with subleading functional weight are not seen by the symbol, they can be constrained by symmetry requirements. Firstly, MHV amplitudes are invariant under parity (spatial reflection), which is realized by $w_{i} \leftrightarrow \bar{w}_{i}$ in the multi-Regge limit [48], that is $x \leftrightarrow \bar{x}$ and $y \leftrightarrow \bar{y}$. Secondly, the multi-Regge limit amplitude should be invariant under target-projectile symmetry (exchange of the two ingoing momenta), which amounts to symmetry under $w_{5} \leftrightarrow 1 / w_{6}$ [58], that is $x \leftrightarrow y$ and $\bar{x} \leftrightarrow \bar{y}$. The sum of six-point terms that is subtracted in the definition (5.4) of the function $g_{3}$ is separately invariant under these transformations, and hence we can require parity as well as target-projectile symmetry for the function $g_{3}$ by itself. These symmetries significantly reduce the number of free parameters in the components of $g_{3}$, as summarized in table 1 . In particular, target-projectile symmetry also swaps $\varepsilon_{5}$ and $\varepsilon_{6}$, such that it fixes $g_{3}^{0,1}$ and $h_{3}^{0,1}$ uniquely in terms of $g_{3}^{1,0}$ and $h_{3}^{1,0}$.

Both parity and target projectile symmetry are not trivially implemented: the parity map replaces all holomorphic weight vectors and arguments of our single-valued basis functions $G^{\mathbf{s}}$ with their complex conjugates. Again using the antipode, these conjugate singlevalued functions can be re-expressed in terms of single-valued functions with holomorphic 
arguments [33], but those will not necessarily be elements of the basis (7.7). Similarly, the target-projectile inversion map $x \leftrightarrow y$ produces non-basis functions. In order to derive constraints for our ansatz coefficients, all non-basis functions need to be re-expressed in terms of basis functions, which is possible due to the many relations among multiple polylogarithms, such as shuffle and stuffle algebra relations. The single-valued map [33] is an algebra homomorphism, hence every identity among ordinary multiple polylogarithms lifts to a corresponding identity among single-valued multiple polylogarithms. In this way, single-valued multiple polylogarithms inherit the shuffle and stuffle algebra relations from their ordinary counterparts, as well as the simpler rescaling property

$$
G^{\mathbf{s}}\left(a_{1}, \ldots, a_{n} ; z\right)=G^{\mathbf{s}}\left(c a_{1}, \ldots, c a_{n} ; c z\right) \quad \text { for } a_{n} \neq 0 \text { and } c \neq 0 .
$$

While one can relate non-basis functions back to basis functions by suitably combining the right shuffle and stuffle identities, it is often more direct to just match a non-basis function to a combination of basis functions using numerics, at least up to the relatively low weight that we consider here. For example, all multiple polylogarithms up to weight four can be expressed in terms of classical polylogarithms $\mathrm{Li}_{m}(z)$ as well as $\operatorname{Li}_{2,2}(z)$ using e.g. the Mathematica package provided by [59]. Since classical polylogarithms can be readily evalueated numerically, it is straightforward to match all non-basis functions up to weight four against combinations of basis functions and zeta values. However, numerics beyond weight four are not readily available, and thus implementing target-projectile symmetry for $g_{3, \mathrm{R}}^{0,0}$ would require to compile all function identities at weight five by algebraic means. We have not attempted to do so, as it is rather laborious, and looking at table 1, targetprojectile symmetry for $g_{3, \mathrm{R}}^{0,0}$ would yield around $\sim 15$ more constraints, which would not get us significantly closer to determining the function $g_{3}$ completely. Parity invariance is less demanding in that regard, as the only functions that cannot be related back to basis functions by simple shuffle algebra relations are harmonic polylogarithms of weight four or less. In appendix C, we list some of the relations among single-valued polylogarithms that are needed to evaluate parity and target-projectile symmetry, and we provide all further relations in the supplementary material.

Collinear limit. Another set of constraints comes from the expansion around the collinear limit. Since the BDS amplitude correctly captures the leading behavior in the collinear limit in the Mandelstam regions that we consider, the remainder function has to vanish in this limit. In order to take the collinear limit, we map our variables $(x, \bar{x})$ and $(y, \bar{y})$ back to $F_{5,6}$ and $r_{5,6}$ via $(7.3)$ and $(6.4)$, which gives

$$
\begin{array}{ll}
x=-\frac{F_{5} r_{5}\left(1+F_{6} r_{6}\right)}{F_{6} r_{6}}, & \bar{x}=-r_{5}\left(F_{6}+r_{6}\right) F_{5} r_{6}, \\
y=-\frac{F_{6}\left(1+F_{5} r_{5}\right) r_{6}}{F_{5} r_{5}}, & \bar{y}=-\frac{\left(F_{5}+r_{5}\right) r_{6}}{F_{6} r_{5}} .
\end{array}
$$

While the Regge limit sits at $T_{j} \rightarrow 0, S_{j} \rightarrow \infty$ with $r_{j}=1 /\left(S_{j} T_{j}\right)$ fixed, the collinear limit is defined by $T_{j} \rightarrow 0$ with $S_{j}$ finite. From the Regge limit, the combined Regge-collinear limit is therefore attained by letting $r_{j} \rightarrow \infty$, that is

$$
x \approx-F_{5} r_{5} \rightarrow-\infty, \quad y \approx-F_{6} r_{6} \rightarrow-\infty .
$$


In this limit, the harmonic polylogarithm part

$$
\left\{G(\vec{a}, x) \mid a_{i} \in\{0,1\}\right\} \cup\{\text { c.c. }\},
$$

of the basis (7.5) expands into logarithms and inverse powers of $x$ (and $\bar{x}$ ). For the other part of the basis:

$$
\left\{G(\vec{a}, 1 / y) \mid a_{i} \in\{0,1, x\}\right\} \cup\{\text { c.c. }\},
$$

the expansion is even simpler, since the argument $1 / y$ tends to zero, while the weights $x$ tend to infinity. The basis functions expand to

$$
\begin{aligned}
G(0,1 / y) & =\log (1 / y) \\
G(0, \ldots, 0,1,1 / y) & =-1 / y+\mathcal{O}\left(1 / y^{2}\right), \\
G(0, \ldots, 0, x, 1 / y) & =-1 / x y+\mathcal{O}\left(1 / y^{2}\right),
\end{aligned}
$$

and all functions $G(\ldots, 1 / y)$ with more than two non-zero weights are of order $\mathcal{O}\left(1 / y^{2}\right)$. After writing all single-valued functions $G^{\mathbf{s}}$ in terms of ordinary multiple polylogarithms and applying the above, we obtain the expansions of the ansätze near the collinear limit (7.12). In doing so, one has to be careful in picking consistent branches for all occurring logarithms. Every single-valued multiple polylogarithm expands to a power series in $\log \left(r_{i}\right)$ and $1 / r_{i}$, where the series coefficients are rational functions of $F_{5}$ and $F_{6}$ as well as zeta values.

The first constraint comes from the fact that the remainder function should vanish in the collinear limit, that is there should be no terms that are free of $1 / r_{i}$ factors. This already implies 37 further constraints on the ansatz, as can be seen in the fifth line in table 1. Moreover, we can require consistency with the general form of the Wilson loop OPE that governs the remainder function in the collinear limit $[17,18]$. The general systematics of the Wilson loop OPE predicts that the remainder function in the combined Regge-collinear limit (at three loops and in any kinematic region) takes the form

$$
\begin{aligned}
R_{7}^{\mathrm{MRL}-\mathrm{coll}}= & \frac{\cos \left(\phi_{5}\right)}{r_{5}} f_{5}\left(\log \left(\varepsilon_{6}\right), \log \left(r_{5}\right)\right)+\frac{\cos \left(\phi_{6}\right)}{r_{6}} f_{6}\left(\log \left(\varepsilon_{5}\right), \log \left(r_{6}\right)\right) \\
& +\frac{\cos \left(\phi_{5}+\phi_{6}\right)}{r_{5} r_{6}} h\left(\log \left(\varepsilon_{5}\right), \log \left(\varepsilon_{6}\right), \log \left(r_{5}\right), \log \left(r_{6}\right)\right) \\
& +\frac{\cos \left(\phi_{5}-\phi_{6}\right)}{r_{5} r_{6}} \bar{h}\left(\log \left(\varepsilon_{5}\right), \log \left(\varepsilon_{6}\right), \log \left(r_{5}\right), \log \left(r_{6}\right)\right)+\mathcal{O}\left(r_{5}^{-2}\right)+\mathcal{O}\left(r_{6}^{-2}\right),
\end{aligned}
$$

where $F_{i}=e^{i \phi_{i}}$, and $f_{5}, f_{6}, h$, and $\bar{h}$ are polynomials in the respective logarithms. In particular, the dependence on $\phi_{5}$ and $\phi_{6}$ is very restricted. ${ }^{17} \mathrm{~A}$ general combination of multiple polylogarithms would also produce sine functions of $\phi_{5}, \phi_{6}$, and $\phi_{5} \pm \phi_{6}$. It turns out that our parity and target-projectile symmetric ansatz is already free of such sine terms, which is an important cross-check of our result. Moreover, terms where $\cos \left(\phi_{5}\right)$

\footnotetext{
${ }^{17}$ The form $(7.18)$ is valid in the Euclidean region as well as the $(+++)$ region. During the analytic continuation into the (---) region, all cross ratios $U_{i j}$ follow closed loops with identical start and end points. Moreover, in the Basso-Sever-Vieira expressions for the cross ratios in general kinematics [60], $\phi_{5}$ and $\phi_{6}$ only appear in the combinations $\cos \left(\phi_{5}\right), \cos \left(\phi_{6}\right)$, and $\cos \left(\phi_{5}+\phi_{6}\right)$. The cosine is an entire function, and hence the general form (7.18) is preserved under the analytic continuation into the (---) region.
} 
multiplies $\log \left(\varepsilon_{5}\right)$ or $\log \left(r_{6}\right)$ should be absent, and the same is true for products of $\cos \left(\phi_{6}\right)$ with $\log \left(\varepsilon_{6}\right)$ or with $\log \left(r_{5}\right) .{ }^{18}$ The absence of such terms provides yet more constraints on the coefficients in our ansatz for $g_{3}$, as can be seen in the sixth line in table 1 .

When considering the above constraints, one has to keep in mind that the remainder function in the (---) region consists of the function $g_{3}$ as well as two copies of the six-point $(--)$ region remainder function (5.13). The six-point three-loop remainder function in multi-Regge kinematics has been determined in [20,49]. In principle, there could be crossterms between the six-point functions and the function $g_{3}$, such that only their sum vanishes and satisfies (7.18) in the collinear limit. However, we have checked that all coefficients (LLA, NLLA, and NNLLA, real and imaginary parts) of the six-point function separately vanish and satisfy (7.18) in the seven-point Regge-collinear limit, for both arguments $v_{5}=$ $-x$ and $v_{6}=-y$. Hence also $g_{3}$ has to satisfy these constraints by itself.

In fact, the Regge-collinear limit is not unique: by cyclically rotating the tessellation of the heptagon that defines the OPE variables (6.2) and taking appropriate limits in the variables $S_{i}$, we can probe different limits in the space of multi-Regge kinematics. Not all collinear limits have an overlap with the multi-Regge limit: the requirement is that the vanishing of "small" cross ratios $u_{j, 2}, u_{j, 3}$ is compatible with the collinear limit $T_{5}, T_{6} \rightarrow 0$. One further case where this is satisfied is the cyclic rotation of the Basso-Sever-Vieira variables by 4 sites, that is we use the momentum twistors $Z_{i}^{\text {here }}=Z_{i+4}^{\mathrm{BSV}}$, where $Z_{i}^{\mathrm{BSV}}$ are defined in appendix A of [18]. ${ }^{19}$ In this case, the collinear-Regge limit is attained by setting $S_{5}=r_{5} T_{5}, S_{6}=1 /\left(r_{6} T_{6}\right)$, and letting $T_{5}, T_{6} \rightarrow 0$. The multi-Regge parameters $w_{5}$, $w_{6}$ are then related to the OPE variables by

$$
r_{5}^{2}=\frac{1}{w_{5} \bar{w}_{5}}, \quad r_{6}^{2}=\frac{1}{w_{6} \bar{w}_{6}}, \quad F_{5}^{2}=\frac{w_{5}}{\bar{w}_{5}}, \quad F_{6}^{2}=\frac{w_{6}}{\bar{w}_{6}},
$$

which implies

$$
x=-\frac{F_{6}\left(F_{5}+r_{5}\right)}{r_{5} r_{6}}, \quad y=-\frac{r_{5}\left(F_{6}+r_{6}\right)}{F_{5} F_{6}} .
$$

Conversely, the combined collinear-Regge limit is attained from the multi-Regge limit by inverting (7.19) for $w_{5}, w_{6}$, and letting $r_{5}, r_{6} \rightarrow \infty$, which implies

$$
x \approx-\frac{F_{6}}{r_{6}} \rightarrow 0, \quad y \approx-\frac{r_{5} r_{6}}{F_{5} F_{6}} \rightarrow-\infty .
$$

In this case, the expansion of the basis functions (7.5) is even simpler, since all arguments $x, \bar{x}, 1 / y$, and $1 / \bar{y}$ tend to zero. Expanding the ansätze for our component functions, we can again require (i) vanishing of all components in the collinear limit, and (ii) agreement with the general form (7.18) of the Wilson loop OPE. These constraints further reduce the ansätze by a few parameters, as shown in the last two lines of table 1. It turns out that this second collinear limit does not yield new further constraints at LLA and NLLA. The NNLLA functions on the other hand do get constrained further.

\footnotetext{
${ }^{18}$ Note the flipping of the indices 5 and 6 in (6.4).

${ }^{19}$ In the case considered above, we used $Z_{i}=Z_{i+1}^{\mathrm{BSV}}$.
} 
The final answer. Putting all pieces together, one arrives at the most general combination of multiple polylogarithms that is parity symmetric, target-projectile symmetric, agrees with the symbol of $g_{3}$, vanishes in the collinear limit, and matches the general form of the Wilson loop OPE in the collinear limit. The resulting function is too bulky for display here, but is attached in the Mathematica file g3fctn.m (see supplementary material). It still contains 155 undetermined coefficients, as summarized in table 1. The space of parameters could perhaps be further reduced by matching subleading terms in the expansion in $1 / r_{i}$ around the collinear limit to the predictions from the Wilson loop OPE [18], or by inspecting the double discontinuity of the symbol. We defer a more detailed analysis of these further constraints to future work.

The functions $g_{3}^{1,1}$ and $h_{3}^{1,1}$ constitute the LLA part of the function $g_{3}$, they solely stem from the first diagram in (5.4), and are not affected by the subtraction of the six-point functions $f_{1,(3)}$. We display the full LLA part of $g_{3}$ in appendix D. The NLLA parts $g_{3}^{1,0}$ and $h_{3}^{1,0}$ comprise the diagrams 2,4 , and 7 in (5.4), and the functions $g_{3}^{0,1}$, and $h_{3}^{0,1}$ consist of the diagrams 3,5 , and 6 . Finally, $g_{3}^{0,0}$ and $h_{3}^{0,0}$ constitute the NNLLA part of the function $g_{3}$, and are composed of the last eight diagrams in (5.4). Notably, the NLLA parts $g_{3}^{1,0}$ and $h_{3}^{1,0}$ of transcendental weight four are expressed solely in terms of products of lower-weight functions $G^{\mathbf{s}}$. That is, they are free of weight-four functions $G^{\mathbf{s}}$ that cannot be expressed in terms of lower-weight functions. This is a general feature of single-valued multiple polylogarithms: every real even-weight single-valued multiple polylogarithm is expressible in terms of (products of) lower-weight functions. This property follows from the defining map (7.6) together with the fact that complex conjugation acts on single-valued functions through the antipode map. ${ }^{20}$

\section{Conclusion}

Summary. Exponentiation and factorization are core features of the Regge limit. In the expansion around large logarithms, they admit a reconstruction of perturbative amplitudes to any multiplicity, once the BFKL building blocks (eigenvalues, impact factors, emission blocks) are known. In this work, we have made this reconstruction explicit, up to the three-loop order.

A central result is the relation (5.13), which expresses the simplest cut contribution to the $n$-point remainder function at three loops in terms of a few basic building blocks. It should be emphasized that the identity has a two-fold meaning: on the one hand, it holds at the level of the complete remainder function's symbol. On the other hand, it holds at the level of full functions once one restricts the remainder function to its simplest cut contribution as in (3.5), neglecting the Regge pole terms as well as higher Regge cut contributions such as the ones in the last line of (2.23). The decomposition of the twoReggeon cut contribution into building blocks is closely tied to the map (4.13) between conventional multi-Regge limit variables $w_{i}$ and "building-block variables" $v_{i}$.

The second main result is the determination of the three-loop building block $g_{3}$ at the level of the symbol from the known seven-point three-loop symbol for general kinematics.

\footnotetext{
${ }^{20}$ I thank the JHEP referee for pointing out this fact.
} 
Together with the symbol of the known six-point building block [49], this permits the reconstruction of the three-loop remainder function symbol at leading logarithmic order, as implemented in the attached Mathematica file (see supplementary material).

Finally, we have constructed a function representative for the building block $g_{3}$, based on the knowledge of its symbol as well as the relevant function space, and by imposing further constraints such as parity invariance, target-projectile symmetry, and consistency with the Wilson loop OPE.

Outlook. It would be interesting to better understand the general relation between the BFKL building blocks - impact factors, eigenvalues, and emission blocks — and the perturbative building blocks that we found for the full cut contributions. Of course, this relation is in principle provided by the Fourier-Mellin transform. However, the action of the inverse Fourier-Mellin transform on general expressions of multiple polylogs is (to the author's knowledge) not understood systematically. Especially, it would be interesting to understand how much can be learnt about the BFKL building blocks when the cut contributions are only known at the symbol level. A better understanding of this point would admit to extract the NLO emission block from two-loop data, from which the threeloop NLO building blocks $g_{L}$ and $g_{R}$ could then be constructed.

We have only fully determined the three-loop seven-point building block $g_{3}$ at leading functional transcendental weight. The parts with lower functional weight (which are multiplied by $\pi$ and zeta values) have been constrained by symmetry requirements, but still contain considerable uncertainty in the form of unfixed rational coefficients. It would be desirable to further constrain the space of parameters, for example by a more detailed comparison to the Wilson loop OPE [18]. This would require to explicitly compute the functions $f_{5}, f_{6}, h$, and $\bar{h}$ by taking the relevant discontinuity of the three-loop OPE answer. In fact, Basso, Caron-Huot and Sever could extract the two-particle cut $f_{k,(1)}$ to all loop orders from the six-point Wilson loop OPE by an ingenuous analytic continuation in the spectral parameter plane [52]. Of course, reconstructing the full three-loop cut contribution $f_{k,(3)}$ for any number of points also requires knowledge of the higher building blocks $g_{\mathrm{L}}, g_{\mathrm{R}}$ (at NLLA), and $h$ (at NNLLA). Beyond that, constructing the full multi-Regge limit remainder function at subleading functional transcendentality in all kinematic regions also requires to take more general multi-Reggeon cut terms into account, such as the ones shown in the last line of (2.23). While it is possible to project out these more general cut terms by restricting to kinematic regions where only adjacent momenta have been flipped, these higher cut terms form an interesting subject on their own, and it would be very interesting to understand them systematically.

\section{Acknowledgments}

I sincerely wish to thank Jochen Bartels for many very instructive and enjoyable discussions, as well as for comments on the manuscript. I also want to thank Johannes Brödel, Vsevolod Chestnov, Georgios Papathanasiou, Volker Schomerus, and Martin Sprenger for valuable discussions. My work is supported by a Marie Curie International Outgoing Fellowship 
within the $7^{\text {th }}$ European Community Framework Programme under Grant No. PIOF-GA2011-299865.

\section{A Reduction identities}

Here, we want to derive the reduction identities (4.5). The absorption of adjacent emission blocks into impact factors was demonstrated in [26], and the analysis directly implies the reduction identity for emission blocks alone. We reproduce it here for completeness.

The identities are most easily understood in momentum space. At leading order, the central emission block simply consists of a single effective Reggeon-Reggeon-gluon vertex attached to the upper Reggeon line in the two-Reggeon state, see figure 2. For a produced gluon with definite helicity, this effective vertex equals [61]

$$
\underset{k_{2} \downarrow^{p_{2}} k_{3}}{k^{2}}=-\sqrt{2} \frac{k_{2} \bar{k}_{3}}{\bar{p}_{2}} .
$$

Compared to the full amplitude, the remainder function has the tree amplitude divided out. We are computing cut contributions to the remainder function, hence we need to divide by the tree-level expression for gluon emission

$$
-\sqrt{2} \frac{q_{2} \bar{q}_{3}}{\bar{p}_{2}}
$$

At leading order, the central emission block in momentum space therefore equals

$$
C_{2} \equiv C\left(q_{2}, k_{2}, p_{2}\right)=\frac{k_{2} \bar{k}_{3}}{q_{2} \bar{q}_{3}}=\frac{\bar{k}_{2}\left(k_{2}+p_{2}\right)}{\bar{q}_{2}\left(q_{2}+p_{2}\right)} .
$$

Combining two leading-order emission blocks requires to include the intermediate transverse propagator $1 /\left|k_{3}\right|^{2}$, again divided by the corresponding tree-level expression $1 /\left|q_{3}\right|^{2}$ :

$$
C_{2} \cdot \frac{\left|q_{3}\right|^{2}}{\left|k_{3}\right|^{2}} \cdot C_{3}=\frac{k_{2} \bar{k}_{3}}{q_{1} \bar{q}_{3}} \cdot \frac{\left|q_{3}\right|^{2}}{\left|k_{3}\right|^{2}} \cdot \frac{k_{3} \bar{k}_{4}}{q_{3} \bar{q}_{4}}=\frac{k_{2} \bar{k}_{4}}{q_{2} \bar{q}_{4}}=\frac{\bar{k}_{2}\left(k_{2}+p_{2}+p_{3}\right)}{\bar{q}_{2}\left(q_{2}+p_{2}+p_{3}\right)} .
$$

This clearly equals the single emission block (A.3) with the emitted momentum $p_{2}$ replaced by the sum of momenta $p_{2}+p_{3}$. Iterating the procedure straightforwardly yields the reduction identity for emission blocks, on the right in (4.5).

The leading-order impact factor consists of a single gluon emission from the bottom Reggeon line, as in figure 2. It thus reads [10]

$$
-\sqrt{2} \frac{q_{1}\left(\bar{q}_{2}-\bar{k}_{2}\right)}{\bar{k}^{\prime}}=-\sqrt{2} \frac{q_{1} \bar{q}_{2}}{\bar{p}_{1}}-\sqrt{2} \frac{\left|q_{1}\right|^{2} \bar{k}_{2}}{\bar{k}^{\prime} \bar{p}_{1}} .
$$

On the right, the emission factor has been split into a "local" piece (first term) and a "nonlocal" part (second term). The local piece plays a role for the one-loop amplitude, but does not affect the remainder function $[10,26]$. It therefore can be dropped for the purpose of computing discontinuities of the remainder function. Thus only the second term remains for the leading-order impact factor. Dividing by the tree expression $-\sqrt{2} q_{1} \bar{q}_{2} / \bar{p}_{1}$, it becomes

$$
\Phi_{\mathrm{L}, 1} \equiv \Phi_{\mathrm{L}}\left(q_{1}, k^{\prime}, p_{1}\right)=\frac{\bar{q}_{1} \bar{k}_{2}}{\bar{k}^{\prime} \bar{q}_{2}}=\frac{\bar{q}_{1}\left(\bar{k}^{\prime}+\bar{p}_{1}\right)}{\bar{k}^{\prime}\left(\bar{q}_{1}+\bar{p}_{1}\right)} .
$$




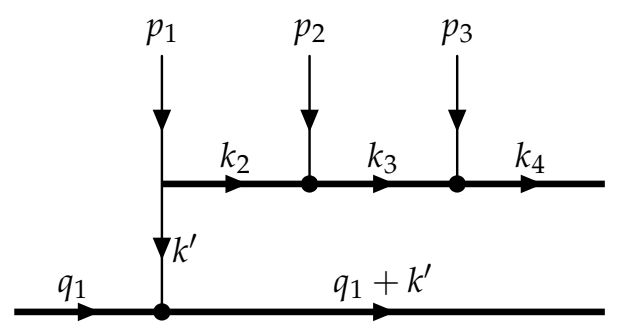

Figure 2. Leading-order impact factor and emission vertices. The total $t$-channel momenta are denoted $q_{i}$ (see figure 1), hence the momentum on the lower Reggeon line equals $q_{1}+k^{\prime}=q_{2}-k_{2}=$ $q_{3}-k_{3}=q_{4}-k_{4}$.

Combining this impact factor with an adjacent emission block, one again needs to include the intermediate propagator factor, which yields

$$
\Phi_{\mathrm{L}, 1} \cdot \frac{\left|q_{2}\right|^{2}}{\left|k_{2}\right|^{2}} \cdot C_{2}=\frac{\bar{q}_{1} \bar{k}_{2}}{\bar{k}^{\prime} \bar{q}_{2}} \cdot \frac{\left|q_{2}\right|^{2}}{\left|k_{2}\right|^{2}} \cdot \frac{k_{2} \bar{k}_{3}}{q_{2} \bar{q}_{3}}=\frac{\bar{q}_{1} \bar{k}_{3}}{\bar{k}^{\prime} \bar{q}_{3}}=\frac{\bar{q}_{1}\left(\bar{k}^{\prime}+\bar{p}_{1}+\bar{p}_{2}\right)}{\bar{k}^{\prime}\left(\bar{q}_{1}+\bar{p}_{1}+\bar{p}_{2}\right)} .
$$

This equals the original impact factor (A.6) with the emitted momentum $p_{1}$ replaced by the sum of momenta $p_{1}+p_{2}$. Again, iterating the procedure yields the reduction identity for impact factors, on the left in (4.5).

\section{B Four-loop expansion}

At four loops, the two-Reggeon cut at LLA evidently expands to a sum of six-point, sevenpoint, and eight-point functions:

$$
\begin{aligned}
f_{k,(4)}= & \sum_{j=5}^{k+4} f_{1,(4)}\left(\varepsilon_{j} ; v_{5, k+4 ; j}\right)+\sum_{i=5}^{k+3} \sum_{j=i+1}^{k+4} g_{2,(4)}\left(\varepsilon_{i}, \varepsilon_{j} ; v_{5, j-1 ; i}, v_{i+1, k+4 ; j}\right) \\
& +\sum_{i=5}^{k+2} \sum_{j=i+1}^{k+3} \sum_{m=j+1}^{k+4} g_{3,(4)}\left(\varepsilon_{i}, \varepsilon_{j}, \varepsilon_{m} ; v_{5, j-1 ; i}, v_{i+1, m-1 ; j}, v_{j+1, k+4 ; m}\right)+\mathcal{O}(\mathrm{NLLA}),
\end{aligned}
$$

where

$$
g_{2,(4)}\left(\varepsilon_{5}, \varepsilon_{6} ; w_{5}, w_{6}\right) \equiv f_{2,(4)}\left(\varepsilon_{5}, \varepsilon_{6} ; w_{5}, w_{6}\right)-f_{1,(4)}\left(\varepsilon_{5} ; v_{5,6 ; 5}\right)-f_{1,(4)}\left(\varepsilon_{6} ; v_{5,6 ; 6}\right),
$$

and

$$
\begin{aligned}
g_{3,(4)} & \left(\varepsilon_{5}, \varepsilon_{6}, \varepsilon_{7} ; w_{5}, w_{6}, w_{7}\right) \equiv \\
\equiv & f_{3,(4)}\left(\varepsilon_{5}, \varepsilon_{6}, \varepsilon_{7} ; w_{5}, w_{6}, w_{7}\right)-f_{1,(4)}\left(\varepsilon_{5} ; v_{5,7 ; 5}\right)-f_{1,(4)}\left(\varepsilon_{6} ; v_{5,7 ; 6}\right)-f_{1,(4)}\left(\varepsilon_{7} ; v_{5,7 ; 7}\right) \\
& -g_{2,(4)}\left(\varepsilon_{5}, \varepsilon_{6} ; w_{5}, v_{6,7 ; 6}\right)-g_{2,(4)}\left(\varepsilon_{5}, \varepsilon_{7} ; v_{5,6 ; 5}, v_{6,7 ; 7}\right)-g_{2,(4)}\left(\varepsilon_{6}, \varepsilon_{7} ; v_{5,6 ; 6}, w_{7}\right) .
\end{aligned}
$$

At NLLA, there are three more building blocks that all stem from nine-point data:

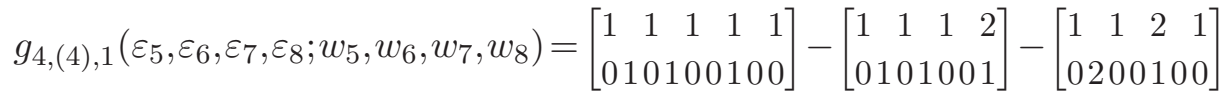

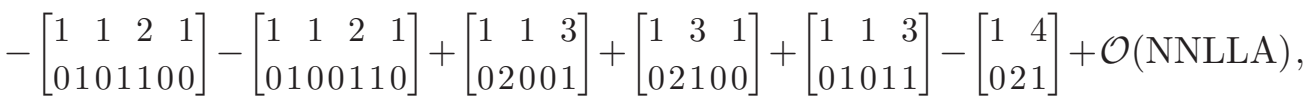




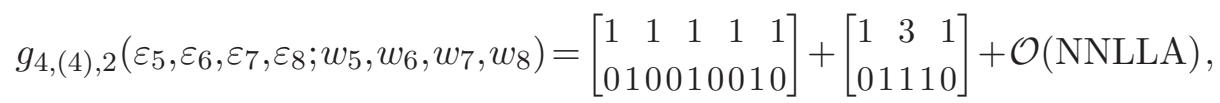

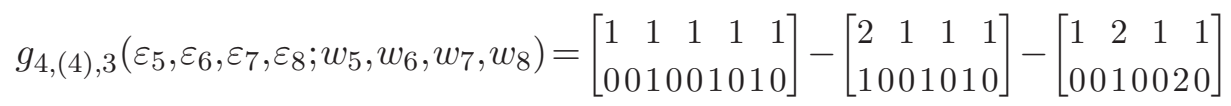

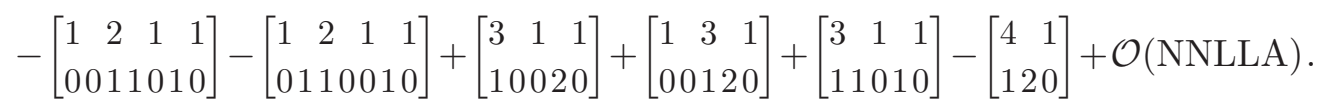

Here, each bracket stands for a BFKL diagram, with the following notation: in the bottom sequence, the numbers alternatingly stand for impact factors / emission blocks and BFKL Green's functions. For the impact factors and emission blocks, the number specifies the loop order. For the Green's functions, it specifies the number of (leading order) BFKL eigenvalues, accompanied by the respective large $\operatorname{logarithm} \log \left(\varepsilon_{j}\right)$; i.e. the numbers 1,2 , and 3 stand for the first, second, and third terms in (5.2). The numbers in the top row specify how many momenta are attached to the respective impact factor or emission block. The expansion (B.1) of the two-Reggeon cut extends to

$$
\begin{aligned}
f_{k,(4)}= & f_{1,(4)}^{\left\{i_{1}, i_{2}\right\}}+g_{2,(4)}^{\left\{i_{1}, i_{2}, i_{3}\right\}}+g_{3,(4)}^{\left\{i_{1}, i_{2}, i_{3}, i_{4}\right\}} \\
& +g_{4,(4), 1}^{\left\{i_{1}, i_{2}, i_{3}, 1, i_{4}\right\}}+g_{4,(4), 2}^{\left\{i_{1}, i_{2}, 1, i_{3}, i_{4}\right\}}+g_{4,(4), 3}^{\left\{i_{1}, 1, i_{2}, i_{3}, i_{4}\right\}}+\mathcal{O}(\text { NNLLA }) .
\end{aligned}
$$

Here, each term stands for a sum over partitions $\left\{i_{1}, i_{2}, \ldots\right\}, \sum_{j} i_{j}=k+1$, of the external momenta $p_{4}, \ldots, p_{k+4}$ into subsequences $\left(p_{4}, \ldots, p_{3+i_{1}}\right),\left(p_{4+i_{1}}, \ldots, p_{3+i_{1}+i_{2}}\right), \ldots$, whose sums get attached to the momentum slots of the respective building block. For example,

$$
\begin{aligned}
& g_{4,(4), 1}^{\left\{i_{1}, i_{2}, i_{3}, 1, i_{4}\right\}} \equiv \\
& \quad \equiv \sum_{i=5}^{k+1} \sum_{j=i+1}^{k+2} \sum_{m=j+2}^{k+4} g_{4,(4), 1}\left(\varepsilon_{i}, \varepsilon_{j}, \varepsilon_{m-1}, \varepsilon_{m} ; v_{5, j-1 ; i}, v_{i+1, m-2 ; j}, v_{j+1, m-1 ; m-1}, v_{m, k+4 ; m}\right) .
\end{aligned}
$$

At NNLLA and NNNLLA, there are many more terms and building blocks. The complete expansion of the two-Reggeon cut reads

$$
\begin{aligned}
f_{k,(4)}= & f_{1,(4)}^{\left\{i_{1}, i_{2}\right\}}+g_{2,(4)}^{\left\{i_{1}, i_{2}, i_{3}\right\}}+g_{3,(4)}^{\left\{i_{1}, i_{2}, i_{3}, i_{4}\right\}}+g_{4,(4), 1}^{\left\{i_{1}, i_{2}, i_{3}, 1, i_{4}\right\}}+g_{4,(4), 2}^{\left\{i_{1}, i_{2}, 1, i_{3}, i_{4}\right\}}+g_{4,(4), 3}^{\left\{i_{1}, 1, i_{2}, i_{3}, i_{4}\right\}} \\
& +g_{4,(4), 4}^{\left\{1, i_{1}, 1, i_{2}, i_{3}\right\}}+g_{4,(4), 5}^{\left\{1, i_{1}, i_{2}, 1, i_{3}\right\}}+g_{4,(4), 6}^{\left\{i_{1}, 1,1, i_{2}, i_{3}\right\}}+g_{4,(4), 7}^{\left\{i_{1}, 1, i_{2}, 1, i_{3}\right\}}+g_{4,(4), 8}^{\left\{i_{1}, 1, i_{2}, i_{3}, 1\right\}} \\
& +g_{4,(4), 9}^{\left\{i_{1}, i_{2}, 1,1, i_{3}\right\}}+g_{4,(4), 10}^{\left\{i_{1}, i_{2}, 1, i_{3}, 1\right\}}+g_{5,(4), 1}^{\left\{i_{1}, 1, i_{2}, 1, i_{3}, i_{4}\right\}}+g_{5,(4), 2}^{\left\{i_{1}, 1, i_{2}, i_{3}, 1, i_{4}\right\}}+g_{5,(4), 3}^{\left\{i_{1}, i_{2}, 1, i_{3}, 1, i_{4}\right\}} \\
& +g_{4,(4), 11}^{\left\{i_{1}, 1, i_{2}, 1,1\right\}}+g_{4,(4), 12}^{\left\{i_{1}, 1,1, i_{2}, 1\right\}}+g_{4,(4), 13}^{\left\{i_{1}, 1,1,1, i_{2}\right\}}+g_{4,(4), 14}^{\left\{1, i_{1}, 1, i_{2}, 1\right\}}+g_{4,(4), 15}^{\left\{1, i_{1}, 1,1, i_{2}\right\}}+g_{4,(4), 16}^{\left\{1,1, i_{1}, 1, i_{2}\right\}} \\
& +g_{5,(4), 4}^{\left\{i_{1}, 1, i_{2}, 1, i_{3}, 1\right\}}+g_{5,(4), 5}^{\left\{i_{1}, 1, i_{2}, 1,1, i_{3}\right\}}+g_{5,(4), 6}^{\left\{i_{1}, 1,1, i_{2}, 1, i_{3}\right\}}+g_{5,(4), 7}^{\left\{1, i_{1}, 1, i_{2}, 1, i_{3}\right\}}+g_{6,(4)}^{\left\{i_{1}, 1, i_{2}, 1, i_{3}, 1, i_{4}\right\}}
\end{aligned}
$$

Terms in the second and third lines start at $\mathcal{O}$ (NNLLA), terms in the last two lines are of order $\mathcal{O}$ (NNNLLA). The individual terms are listed explicitly in the following. The completion of the NLLA terms (B.4)-(B.6) is given by

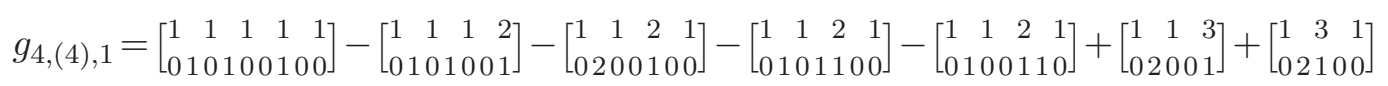

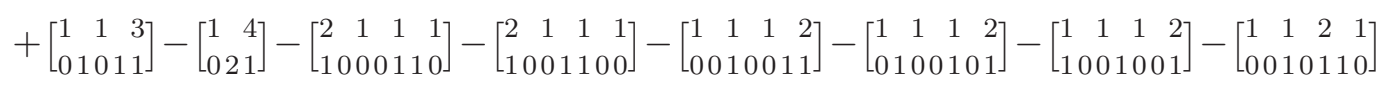




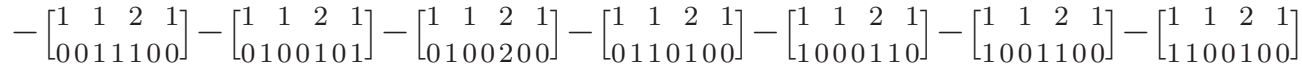

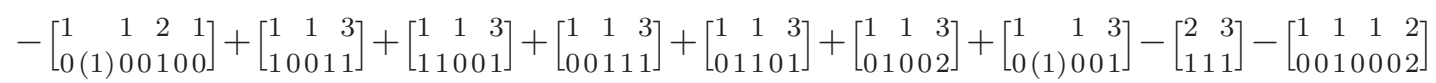

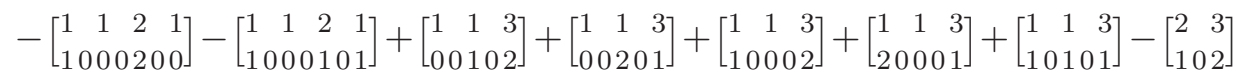

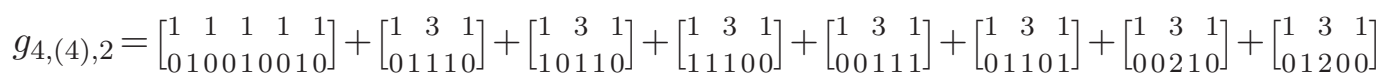

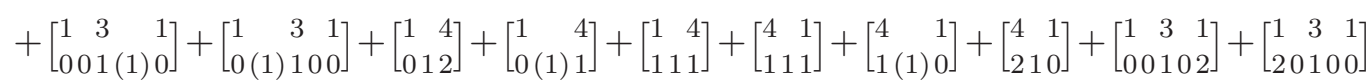

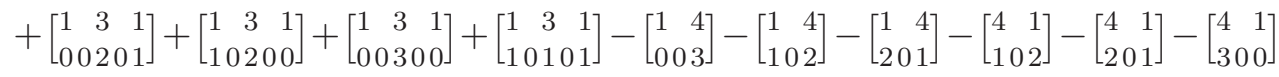

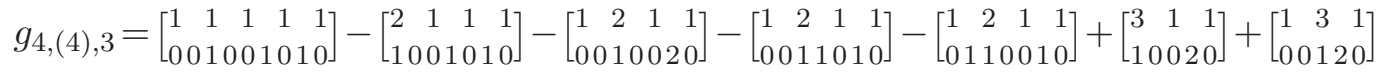

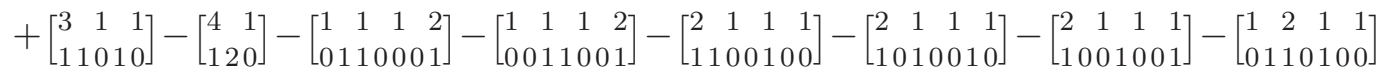

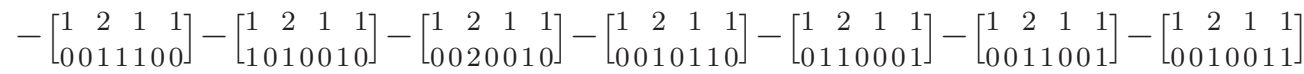

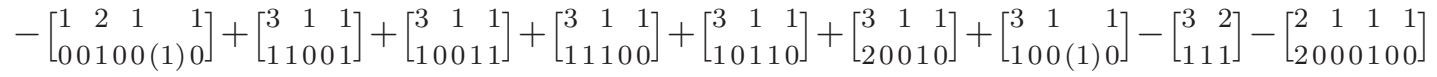

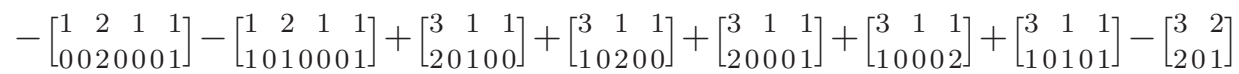

Here, a (1) in the place of a Green's function stands for the one-loop correction to the BFKL eigenvalue, i.e. for the fourth term in (5.2). The following terms start at $\mathcal{O}$ (NNLLA):

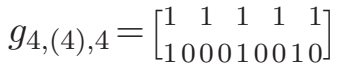

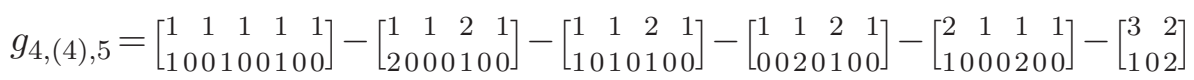

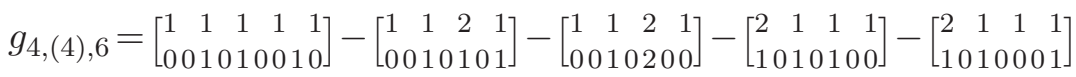

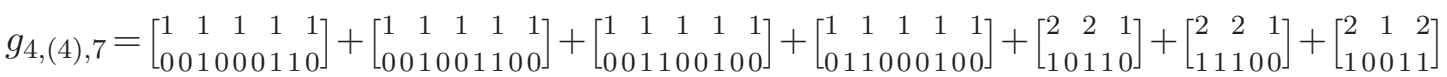

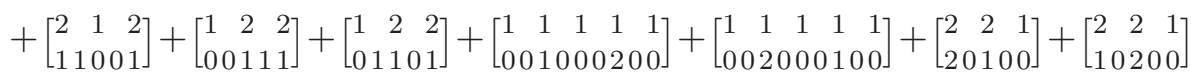

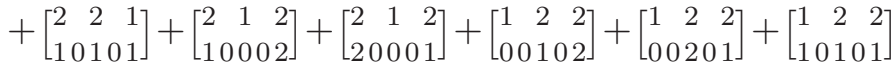

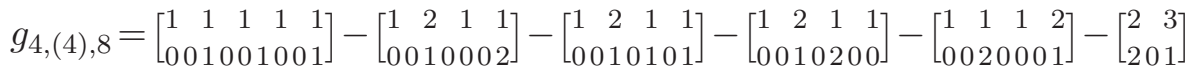

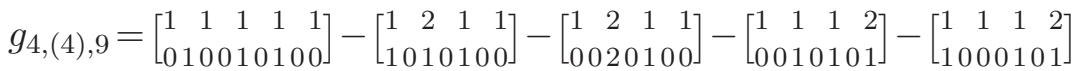

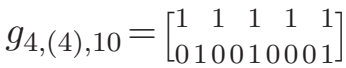

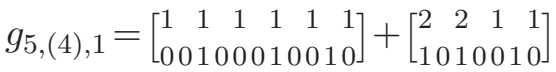

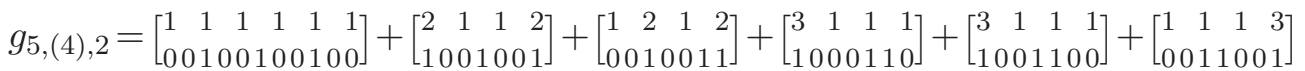




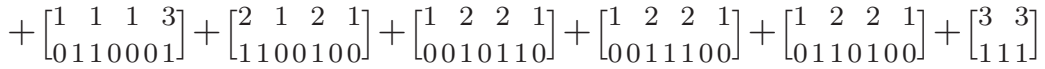

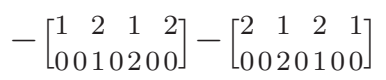

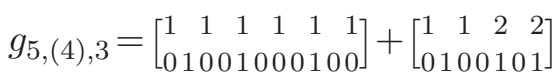

Finally, the following terms only contribute at NNNLLA:

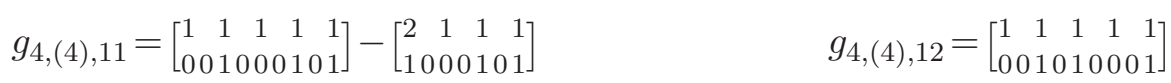

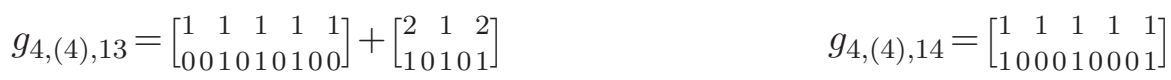

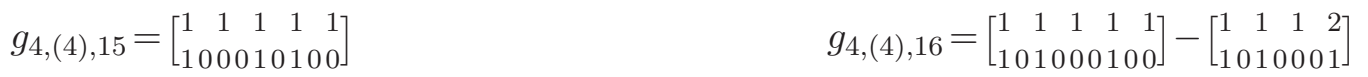

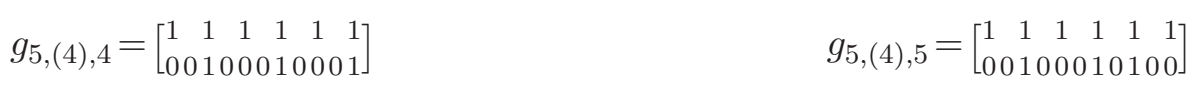

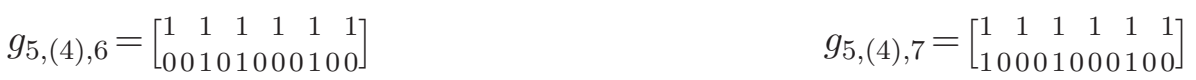

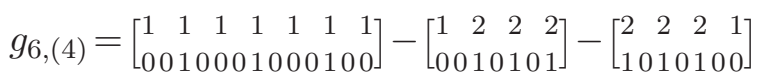

The large number of terms at NNLLA and NNNLLA shows that the decomposition into building blocks is less effective than at two and three loops. The reason is that the reduction identities (4.5) are only established for the leading order emission block. It would be interesting to see whether the (thus far unknown) NLO emission block satisfies similar reduction identities, in which case many of the above terms could be reduced and absorbed into a smaller number of building blocks, reducing the complexity of the decomposition.

\section{Polylogarithm identities}

When applying the target-projectile transformation $x \leftrightarrow y$ or the parity map $x \rightarrow \bar{x}$, $y \rightarrow \bar{y}$ to the ansatz functions for the components of $g_{3}$, some single-valued basis functions are mapped to non-basis single-valued multiple polylogarithms. In order to impose the required symmetries on the ansätze, these non-basis functions need to be re-expressed in terms of basis functions. This can always be achieved with the help of shuffle and stuffle relations, as well as the rescaling property (7.9). Shuffle relations take the form

$$
G(\vec{a} ; z) G(\vec{b} ; z)=\sum_{\vec{c} \in \vec{a} \Perp \vec{b}} G(\vec{c} ; z),
$$

where the sum runs over all shuffles $\vec{a} \uplus \vec{b}$ of the weight vectors $\vec{a}$ and $\vec{b}$, that is over all permutations of their components that preserve the ordering of elements within both $\vec{a}$ and $\vec{b}$. The shuffle relation follows directly from the iterated integral definition of multiple polylogarithms (7.2), and they hold for ordinary as well as single-valued multiple polylogarithms. Stuffle relations on the other hand are less transparent in the integral representation; they follow directly from the series representation of $G(\vec{a} ; z)$ around $z=0$, see for example [47]. 
At weights one and two, the following identities are needed to evaluate the parity and target-projectile invariance conditions:

$$
\begin{aligned}
& G_{0}^{\mathbf{s}, y}=-G_{0}^{\mathbf{s}, \check{y}}, \quad G_{0}^{\mathbf{s}, \check{x}}=-G_{0}^{\mathbf{s}, x}, \quad G_{x}^{\mathbf{s}, 1}=-G_{0}^{\mathbf{s}, x}+G_{1}^{\mathbf{s}, x}, \\
& G_{x \check{y}}^{\mathbf{s}, x}=-G_{0}^{\mathbf{s}, \check{y}}+G_{1}^{\mathbf{s}, \check{y}}, \quad G_{x \check{y}}^{\mathbf{s}, \check{y}}=-G_{0}^{\mathbf{s}, x}+G_{1}^{\mathbf{s}, x}, \quad G_{0, x}^{\mathbf{s}, 1}=\frac{1}{2}\left(G_{0}^{\mathbf{s}, x}\right)^{2}-G_{0,1}^{\mathbf{s}, x}, \\
& G_{0, x \check{y}}^{\mathbf{s}, x}=\frac{1}{2}\left(G_{0}^{\mathbf{s}, \check{y}}\right)^{2}-G_{0,1}^{\mathbf{s}, \check{y}}, \quad G_{0, x \check{y}}^{\mathbf{s}, \check{y}}=\frac{1}{2}\left(G_{0}^{\mathbf{s}, x}\right)^{2}-G_{0,1}^{\mathbf{s}, x}, \\
& G_{x \check{y}, x}^{\mathbf{s}, \check{y}}=-G_{0}^{\mathbf{s}, x} G_{1}^{\mathbf{s}, \check{y}}+G_{1}^{\mathbf{s}, x} G_{1}^{\mathbf{s}, \check{y}}+G_{0, x}^{\mathbf{s}, \check{y}}-G_{1, x}^{\mathbf{s}, \check{y}},
\end{aligned}
$$

Here and in the following, we use the condensed notation $G_{a_{1}, \ldots, a_{n}}^{\mathbf{s}, z} \equiv G^{\mathbf{s}}\left(a_{1}, \ldots, a_{n} ; z\right)$, and $\check{y} \equiv 1 / y$. At weight three, we have for example the following identities:

$$
\begin{aligned}
& G_{0,0, x \check{y}}^{\mathbf{s}, x}=-\frac{1}{6}\left(G_{0}^{\mathbf{s}, \check{y}}\right)^{3}+G_{0,0,1}^{\mathbf{s}, \check{y}}, \\
& G_{0, x \check{y}, x \check{y}}^{\mathbf{s}, x}=-\frac{1}{6}\left(G_{0}^{\mathbf{s}, \check{y}}\right)^{3}+G_{0}^{\mathbf{s}, \check{y}} G_{0,1}^{\mathbf{s}, \check{y}}-G_{0,0,1}^{\mathbf{s} \check{y}}-G_{0,1,1}^{\mathbf{s}, \check{y}}+2 \zeta_{3}, \\
& G_{0,0, x \check{y}}^{\mathbf{s}, \check{y}}=-\frac{1}{6}\left(G_{0}^{\mathbf{s}, x}\right)^{3}+G_{0,0,1}^{\mathbf{s}, x}, \\
& G_{0, x, x \check{y}}^{\mathbf{s}, \check{y}}=-\frac{1}{2}\left(G_{0}^{\mathbf{s}, x}\right)^{2} G_{1}^{\mathbf{s}, \check{y}}+G_{1}^{\mathbf{s}, \check{y}} G_{0,1}^{\mathbf{s}, x}+G_{0}^{\mathbf{s}, x} G_{0,1}^{\mathbf{s}, \check{y}}-G_{1}^{\mathbf{s}, x} G_{0,1}^{\mathbf{s}, \check{y}}-G_{0}^{\mathbf{s}, x} G_{0, x}^{\mathbf{s}, \check{y}}+G_{1}^{\mathbf{s}, x} G_{0, x}^{\mathbf{s}, \check{y}} \\
& +G_{1}^{\mathbf{s}, \breve{y}} G_{0, x}^{\mathbf{s}, \breve{y}}-2 G_{0,0, x}^{\mathbf{s}, \breve{y}}-G_{0, x, 1}^{\mathbf{s}, \breve{y}}, \\
& G_{0, x \check{y}, x}^{\mathbf{s}, \check{y}}=\frac{1}{2}\left(G_{0}^{\mathbf{s}, x}\right)^{2} G_{1}^{\mathbf{s}, \check{y}}-G_{1}^{\mathbf{s}, \breve{y}} G_{0,1}^{\mathbf{s}, x}-G_{1}^{\mathbf{s}, \breve{y}} G_{0, x}^{\mathbf{s}, \breve{y}}+G_{0,0, x}^{\mathbf{s}, \check{y}}+G_{0,1, x}^{\mathbf{s}, \breve{y}}+G_{0, x, 1}^{\mathbf{s}, \check{y}}, \\
& G_{0, x \breve{y}, x \check{y}}^{\mathbf{s}, \check{y}}=-\frac{1}{6}\left(G_{0}^{\mathbf{s}, x}\right)^{3}+G_{0}^{\mathbf{s}, x} G_{0,1}^{\mathbf{s}, x}-G_{0,0,1}^{\mathbf{s}, x}-G_{0,1,1}^{\mathbf{s}, x}+2 \zeta_{3}, \\
& G_{x \breve{y}, x, x}^{\mathbf{s}, \check{y}}=-\frac{1}{2} G_{0}^{\mathbf{s}, x}\left(G_{1}^{\mathbf{s}, \check{y}}\right)^{2}+\frac{1}{2} G_{1}^{\mathbf{s}, x}\left(G_{1}^{\mathbf{s}, \check{y}}\right)^{2}+G_{1}^{\mathbf{s}, \check{y}} G_{0, x}^{\mathbf{s}, \breve{y}}-G_{0,1, x}^{\mathbf{s}, \check{y}}-G_{0, x, 1}^{\mathbf{s}, \breve{y}} \\
& +G_{0, x, x}^{\mathbf{s}, \check{y}}-G_{1,1, x}^{\mathbf{s} \check{y}}-G_{1, x, x}^{\mathbf{s}, \check{y}}, \\
& G_{x \breve{y}, x \check{y}, x}^{\mathbf{s}, \check{y}}=\frac{1}{2}\left(G_{0}^{\mathbf{s}, x}\right)^{2} G_{1}^{\mathbf{s}, \breve{y}}-G_{0}^{\mathbf{s}, x} G_{1}^{\mathbf{s}, x} G_{1}^{\mathbf{s}, \breve{y}}+\frac{1}{2}\left(G_{1}^{\mathbf{s}, x}\right)^{2} G_{1}^{\mathbf{s}, \check{y}}+\frac{1}{2} G_{0}^{\mathbf{s}, x}\left(G_{1}^{\mathbf{s}, \check{y}}\right)^{2}-\frac{1}{2} G_{1}^{\mathbf{s}, x}\left(G_{1}^{\mathbf{s}, \check{y}}\right)^{2} \\
& -G_{0}^{\mathbf{s}, x} G_{0,1}^{\mathbf{s}, \check{y}}+G_{1}^{\mathbf{s}, x} G_{0,1}^{\mathbf{s}, \breve{y}}-G_{1}^{\mathbf{s}, \breve{y}} G_{0, x}^{\mathbf{s}, \check{y}}+G_{0,0, x}^{\mathbf{s}, \breve{y}}+G_{0, x, 1}^{\mathbf{s}, \breve{y}}+G_{1,1, x}^{\mathbf{s}, \check{y}} .
\end{aligned}
$$

All of the above equations rely on multiple shuffle and stuffle relations. These are all relations involving both letters $x$ and $y$ that one needs for parity and target-projectile symmetry up to weight four. These as well as all further required relations among single-valued harmonic polylogarithms only involving $x$ can be found in the supplementary material GGtobasis.m.

\section{The function $g_{3}$ at LLA}

For reference, we display the LLA part of the function $g_{3}$, up to a few undetermined coefficients that are not constrained by the symmetries that we considered: ${ }^{21}$

$$
\begin{aligned}
g_{3}^{1,1} & +2 \pi i h_{3}^{1,1}=\frac{1}{16}\left(\left(G_{0}^{\mathbf{s}, x}\right)^{2} G_{1}^{\mathbf{s}, \check{y}}-2 G_{0}^{\mathbf{s}, x} G_{0}^{\mathbf{s}, \check{y}} G_{1}^{\mathbf{s}, \check{y}}+2 G_{0}^{\mathbf{s}, \check{y}} G_{1}^{\mathbf{s}, x} G_{1}^{\mathbf{s}, \check{y}}-\left(G_{1}^{\mathbf{s}, x}\right)^{2} G_{1}^{\mathbf{s}, \check{y}}\right. \\
& +G_{0}^{\mathbf{s}, x}\left(G_{1}^{\mathbf{s}, \check{y}}\right)^{2}-G_{1}^{\mathbf{s}, x}\left(G_{1}^{\mathbf{s}, \check{y}}\right)^{2}+2 G_{0}^{\mathbf{s}, \check{y}} G_{1}^{\mathbf{s}, x} G_{x}^{\mathbf{s}, \check{y}}+\left(G_{1}^{\mathbf{s}, x}\right)^{2} G_{x}^{\mathbf{s}, \check{y}}-2 G_{0}^{\mathbf{s}, \check{y}} G_{1}^{\mathbf{s}, \check{y}} G_{x}^{\mathbf{s}, \check{y}}
\end{aligned}
$$

\footnotetext{
${ }^{21}$ This result agrees with [33] (equation (D.8) there) once we set all parameters $c_{. .}^{\mathrm{i} / \mathrm{r}}$ to zero.
} 


$$
\begin{aligned}
& -2 G_{1}^{\mathbf{s}, x} G_{1}^{\mathbf{s}, \check{y}} G_{x}^{\mathbf{s}, \check{y}}+\left(G_{1}^{\mathbf{s}, \check{y}}\right)^{2} G_{x}^{\mathbf{s}, \check{y}}-2 G_{x}^{\mathbf{s}, \check{y}} G_{0,1}^{\mathbf{s}, x}+2 G_{x}^{\mathbf{s}, \check{y}} G_{0}^{\mathbf{s}, 1, \check{y}}+2 G_{1}^{\mathbf{s}, \check{y}} G_{0, x}^{\mathbf{s}, \check{y}}+2 G_{0}^{\mathbf{s}, x} G_{1}^{\mathbf{s}, x, \check{y}} \\
& \left.-2 G_{1}^{\mathbf{s}, x} G_{1, x}^{\mathbf{s}, \check{y}}+2 G_{1}^{\mathbf{s}, \check{y}} G_{1}^{\mathbf{s}, x, \check{y}}-4 G_{0,1, x}^{\mathbf{s}, \check{y}}-4 G_{0, x, 1}^{\mathbf{s}, \check{y}}-4 G_{1,1, x}^{\mathbf{s}, \check{y}}\right)+c_{2,1}^{\mathrm{r}} G_{x}^{\mathbf{s}, \check{y}} \zeta_{2} \\
& +2 \pi i\left(c_{2,1}^{\mathrm{i}}\left(\left(G_{0}^{\mathbf{s}, x}\right)^{2}-2 G_{0}^{\mathbf{s}, x} G_{1}^{\mathbf{s}, x}+\left(G_{1}^{\mathbf{s}, x}\right)^{2}+\left(G_{1}^{\mathbf{s}, \check{y}}\right)^{2}\right)+c_{2,2}^{\mathrm{i}}\left(G_{0}^{\mathbf{s}, x}-G_{1}^{\mathbf{s}, x}\right) G_{1}^{\mathbf{s}, \check{y}}\right. \\
& \left.+c_{2,3}^{\mathrm{i}}\left(G_{0}^{\mathbf{s}, x}-G_{1}^{\mathbf{s}, x}-G_{1}^{\mathbf{s}, \check{y}}\right) G_{x}^{\mathbf{s}, \check{y}}+c_{2,4}^{\mathrm{i}}\left(G_{0}^{\mathbf{s}, \check{y}}-G_{1}^{\mathbf{s}, x}-G_{1}^{\mathbf{s}, \check{y}}\right) G_{x}^{\mathbf{s}, \check{y}}+c_{2,5}^{\mathrm{i}}\left(G_{x}^{\mathbf{s}, \check{y}}\right)^{2}\right) .
\end{aligned}
$$

Here, we again used the shorthand notation $G_{a_{1}, \ldots, a_{n}}^{\mathbf{s}, z} \equiv G^{\mathbf{s}}\left(a_{1}, \ldots, a_{n} ; z\right)$. The NLLA and NNLLA functions are too lengthy for display, they are attached in the supplementary material g3f cn.m.

Open Access. This article is distributed under the terms of the Creative Commons Attribution License (CC-BY 4.0), which permits any use, distribution and reproduction in any medium, provided the original author(s) and source are credited.

\section{References}

[1] V.S. Fadin, E.A. Kuraev and L.N. Lipatov, On the Pomeranchuk singularity in asymptotically free theories, Phys. Lett. B 60 (1975) 50 [inSPIRE].

[2] E.A. Kuraev, L.N. Lipatov and V.S. Fadin, Multi-reggeon processes in the Yang-Mills theory, Sov. Phys. JETP 44 (1976) 443 [Zh. Eksp. Teor. Fiz. 71 (1976) 840] [INSPIRE].

[3] I.I. Balitsky and L.N. Lipatov, The Pomeranchuk singularity in quantum chromodynamics, Sov. J. Nucl. Phys. 28 (1978) 822 [Yad. Fiz. 28 (1978) 1597] [INSPIRE].

[4] J. Bartels, High-energy behavior in a non-Abelian gauge theory (II), Nucl. Phys. B 175 (1980) 365 [inSPIRE].

[5] J. Kwiecinski and M. Praszalowicz, Three gluon integral equation and odd $C$ singlet Regge singularities in QCD, Phys. Lett. B 94 (1980) 413 [INSPIRE].

[6] N. Beisert et al., Review of AdS/CFT integrability: an overview, Lett. Math. Phys. 99 (2012) 3 [arXiv:1012.3982] [INSPIRE].

[7] Z. Bern, L.J. Dixon and V.A. Smirnov, Iteration of planar amplitudes in maximally supersymmetric Yang-Mills theory at three loops and beyond, Phys. Rev. D 72 (2005) 085001 [hep-th/0505205] [INSPIRE].

[8] L.F. Alday and J. Maldacena, Comments on gluon scattering amplitudes via AdS/CFT, JHEP 11 (2007) 068 [arXiv:0710.1060] [INSPIRE].

[9] J. Bartels, L.N. Lipatov and A. Sabio Vera, BFKL pomeron, reggeized gluons and Bern-Dixon-Smirnov amplitudes, Phys. Rev. D 80 (2009) 045002 [arXiv:0802.2065] [INSPIRE].

[10] J. Bartels, L.N. Lipatov and A. Sabio Vera, $N=4$ supersymmetric Yang-Mills scattering amplitudes at high energies: the Regge cut contribution, Eur. Phys. J. C 65 (2010) 587 [arXiv: 0807.0894] [INSPIRE].

[11] L.N. Lipatov, Analytic properties of high energy production amplitudes in $N=4$ SUSY, Theor. Math. Phys. 170 (2012) 166 [arXiv:1008.1015] [INSPIRE]. 
[12] L.J. Dixon, J.M. Drummond, C. Duhr and J. Pennington, The four-loop remainder function and multi-Regge behavior at NNLLA in planar $N=4$ super-Yang-Mills theory,

JHEP 06 (2014) 116 [arXiv: 1402.3300] [INSPIRE].

[13] L.J. Dixon, M. von Hippel and A.J. McLeod, The four-loop six-gluon NMHV ratio function, JHEP 01 (2016) 053 [arXiv: 1509.08127] [INSPIRE].

[14] A.B. Goncharov, Multiple polylogarithms and mixed Tate motives, math/0103059 [INSPIRE].

[15] F.C.S. Brown, Polylogarithmes multiples uniformes en une variable, Compt. Rend. Math. 338 (2004) 527 [INSPIRE].

[16] S. Caron-Huot and S. He, Jumpstarting the all-loop S-matrix of planar $N=4$ super Yang-Mills, JHEP 07 (2012) 174 [arXiv:1112.1060] [INSPIRE].

[17] B. Basso, A. Sever and P. Vieira, Spacetime and flux tube S-matrices at finite coupling for $N=4$ supersymmetric Yang-Mills theory, Phys. Rev. Lett. 111 (2013) 091602 [arXiv: 1303.1396] [INSPIRE].

[18] B. Basso, A. Sever and P. Vieira, Space-time S-matrix and flux tube S-matrix II. Extracting and matching data, JHEP 01 (2014) 008 [arXiv: 1306. 2058] [INSPIRE].

[19] L.J. Dixon, C. Duhr and J. Pennington, Single-valued harmonic polylogarithms and the multi-Regge limit, JHEP 10 (2012) 074 [arXiv:1207.0186] [INSPIRE].

[20] L.J. Dixon, J.M. Drummond, M. von Hippel and J. Pennington, Hexagon functions and the three-loop remainder function, JHEP 12 (2013) 049 [arXiv:1308.2276] [INSPIRE].

[21] J.M. Drummond and G. Papathanasiou, Hexagon OPE resummation and multi-Regge kinematics, JHEP 02 (2016) 185 [arXiv:1507.08982] [INSPIRE].

[22] J. Broedel and M. Sprenger, Six-point remainder function in multi-Regge-kinematics: an efficient approach in momentum space, JHEP 05 (2016) 055 [arXiv: 1512.04963] [INSPIRE].

[23] J. Broedel, M. Sprenger and A. Torres Orjuela, Towards single-valued polylogarithms in two variables for the seven-point remainder function in multi-Regge-kinematics, Nucl. Phys. B 915 (2017) 394 [arXiv: 1606.08411] [INSPIRE].

[24] J.M. Drummond, G. Papathanasiou and M. Spradlin, A symbol of uniqueness: the cluster bootstrap for the 3-loop MHV heptagon, JHEP 03 (2015) 072 [arXiv:1412.3763] [INSPIRE].

[25] J. Bartels, L.N. Lipatov and A. Prygarin, Collinear and Regge behavior of $2 \rightarrow 4 M H V$ amplitude in $N=4$ super Yang-Mills theory, arXiv:1104.4709 [INSPIRE].

[26] J. Bartels, A. Kormilitzin, L.N. Lipatov and A. Prygarin, BFKL approach and $2 \rightarrow 5$ maximally helicity violating amplitude in $N=4$ super-Yang-Mills theory, Phys. Rev. D 86 (2012) 065026 [arXiv:1112.6366] [INSPIRE].

[27] J. Bartels, A. Kormilitzin and L. Lipatov, Analytic structure of the $n=7$ scattering amplitude in $N=4$ SYM theory in the multi-Regge kinematics: conformal Regge pole contribution, Phys. Rev. D 89 (2014) 065002 [arXiv:1311.2061] [inSPIRE].

[28] J. Bartels, A. Kormilitzin and L.N. Lipatov, Analytic structure of the $n=7$ scattering amplitude in $N=4$ theory in multi-Regge kinematics: conformal Regge cut contribution, Phys. Rev. D 91 (2015) 045005 [arXiv: 1411.2294] [InSPIRE].

[29] J. Bartels, V. Schomerus and M. Sprenger, Multi-Regge limit of the n-gluon bubble ansatz, JHEP 11 (2012) 145 [arXiv:1207.4204] [INSPIRE]. 
[30] J. Bartels, V. Schomerus and M. Sprenger, The Bethe roots of Regge cuts in strongly coupled $N=4$ SYM theory, JHEP 07 (2015) 098 [arXiv:1411.2594] [INSPIRE].

[31] T. Bargheer, G. Papathanasiou and V. Schomerus, The two-loop symbol of all multi-Regge regions, JHEP 05 (2016) 012 [arXiv: 1512.07620] [INSPIRE].

[32] S. Caron-Huot, Superconformal symmetry and two-loop amplitudes in planar $N=4$ super Yang-Mills, JHEP 12 (2011) 066 [arXiv:1105.5606] [INSPIRE].

[33] V. Del Duca et al., Multi-Regge kinematics and the moduli space of Riemann spheres with marked points, JHEP 08 (2016) 152 [arXiv: 1606.08807] [INSPIRE].

[34] T. Regge, Bound states, shadow states and Mandelstam representation, Nuovo Cim. 18 (1960) 947 [inSPIRE].

[35] D. Amati, A. Stanghellini and S. Fubini, Theory of high-energy scattering and multiple production, Nuovo Cim. 26 (1962) 896 [INSPIRE].

[36] S. Mandelstam, Cuts in the angular momentum plane. 2, Nuovo Cim. 30 (1963) 1148 [INSPIRE].

[37] L.N. Lipatov and A. Prygarin, BFKL approach and six-particle $M H V$ amplitude in $N=4$ super Yang-Mills, Phys. Rev. D 83 (2011) 125001 [arXiv:1011.2673] [INSPIRE].

[38] J. Bartels, Analytic properties of the 8-point scattering amplitude: conformal Regge pole and Regge cut contributions, to appear.

[39] The on-line encyclopedia of integer sequences webpage, published electronically, https://oeis.org.

[40] O. Steinmann, Über den Zusammenhang zwischen den Wightmanfunktionen und den retardierten Kommutatoren (in German), Helv. Phys. Acta 33 (1960) 257.

[41] L.N. Lipatov, Integrability of scattering amplitudes in $N=4$ SUSY, J. Phys. A 42 (2009) 304020 [arXiv:0902.1444] [InSPIRE].

[42] S. Caron-Huot and K.J. Larsen, Uniqueness of two-loop master contours, JHEP 10 (2012) 026 [arXiv:1205.0801] [INSPIRE].

[43] N. Arkani-Hamed, J.L. Bourjaily, F. Cachazo, S. Caron-Huot and J. Trnka, The all-loop integrand for scattering amplitudes in planar $N=4$ SYM, JHEP 01 (2011) 041 [arXiv: 1008.2958] [INSPIRE].

[44] N. Arkani-Hamed, J.L. Bourjaily, F. Cachazo and J. Trnka, Local integrals for planar scattering amplitudes, JHEP 06 (2012) 125 [arXiv:1012.6032] [INSPIRE].

[45] A.B. Goncharov, A simple construction of Grassmannian polylogarithms, arXiv:0908.2238 [INSPIRE].

[46] C. Duhr, H. Gangl and J.R. Rhodes, From polygons and symbols to polylogarithmic functions, JHEP 10 (2012) 075 [arXiv:1110.0458] [INSPIRE].

[47] C. Duhr, Mathematical aspects of scattering amplitudes, in Theoretical Advanced Study Institute in elementary particle physics: journeys through the precision frontier: amplitudes for colliders (TASI 2014), Boulder CO U.S.A., 2-27 June 2014 [arXiv:1411.7538] [INSPIRE].

[48] A. Prygarin, M. Spradlin, C. Vergu and A. Volovich, All two-loop MHV amplitudes in multi-Regge kinematics from applied symbology, Phys. Rev. D 85 (2012) 085019 [arXiv:1112.6365] [INSPIRE]. 
[49] L.J. Dixon, J.M. Drummond and J.M. Henn, Bootstrapping the three-loop hexagon, JHEP 11 (2011) 023 [arXiv: 1108.4461] [INSPIRE].

[50] V.S. Fadin and L.N. Lipatov, BFKL equation for the adjoint representation of the gauge group in the next-to-leading approximation at $N=4$ SUSY, Phys. Lett. B 706 (2012) 470 [arXiv:1111.0782] [INSPIRE].

[51] Y. Hatsuda, Wilson loop OPE, analytic continuation and multi-Regge limit, JHEP 10 (2014) 038 [arXiv: 1404.6506] [INSPIRE].

[52] B. Basso, S. Caron-Huot and A. Sever, Adjoint BFKL at finite coupling: a short-cut from the collinear limit, JHEP 01 (2015) 027 [arXiv: 1407.3766] [INSPIRE].

[53] A.V. Kotikov, L.N. Lipatov, A.I. Onishchenko and V.N. Velizhanin, Three loop universal anomalous dimension of the Wilson operators in $N=4$ SUSY Yang-Mills model, Phys. Lett. B 595 (2004) 521 [Erratum ibid. B 632 (2006) 754] [hep-th/0404092] [INSPIRE].

[54] T. Gehrmann and E. Remiddi, Two loop master integrals for $\gamma^{*} \rightarrow 3$ jets: the planar topologies, Nucl. Phys. B 601 (2001) 248 [hep-ph/0008287] [INSPIRE].

[55] D.E. Radford, A natural ring basis for the shuffle algebra and an application to group schemes, J. Alg. 58 (1979) 432.

[56] J. Pennington, The six-point remainder function to all loop orders in the multi-Regge limit, JHEP 01 (2013) 059 [arXiv: 1209. 5357] [INSPIRE].

[57] L.J. Dixon, J.M. Drummond, C. Duhr, M. von Hippel and J. Pennington, Bootstrapping six-gluon scattering in planar $N=4$ super-Yang-Mills theory, in Proceedings, $12^{\text {th }} D E S Y$ Workshop on Elementary Particle Physics: Loops and Legs in Quantum Field Theory (LL2014), Germany, (2014) [PoS (LL2014) 077] [arXiv: 1407.4724] [INSPIRE].

[58] J. Bartels, V. Schomerus and M. Sprenger, Heptagon amplitude in the multi-Regge regime, JHEP 10 (2014) 067 [arXiv: 1405.3658] [INSPIRE].

[59] H. Frellesvig, D. Tommasini and C. Wever, On the reduction of generalized polylogarithms to $L i_{n}$ and $L i_{2,2}$ and on the evaluation thereof, JHEP 03 (2016) 189 [arXiv:1601.02649] [INSPIRE].

[60] B. Basso, J. Caetano, L. Cordova, A. Sever and P. Vieira, OPE for all helicity amplitudes II. Form factors and data analysis, JHEP 12 (2015) 088 [arXiv:1508.02987] [INSPIRE].

[61] L.N. Lipatov, High-energy scattering in $Q C D$ and in quantum gravity and two-dimensional field theories, Nucl. Phys. B 365 (1991) 614 [INSPIRE]. 